\title{
Pseudo-Spectral Control of a Novel Oscillating Surge Wave Energy Converter in Regular Waves, Part I: Power Optimization Including Load Reduction
}

\author{
N. M. Tom ${ }^{\mathrm{a}, *}$, Y. H. Yu ${ }^{\mathrm{a}}$, A. D. Wright ${ }^{\mathrm{a}}$, M. J. Lawson ${ }^{\mathrm{a}}$ \\ ${ }^{a}$ National Renewable Energy Laboratory, MS 3811, 15013 Denver West Parkway, Golden, CO, 80401, USA
}

\begin{abstract}
The aim of this paper is to describe a procedure to maximize the power-to-load ratio of a novel wave energy converter (WEC) that combines an oscillating surge wave energy converter with variable structural components. The control of the power-take-off torque will be on a wave-to-wave timescale, whereas the structure will be controlled statically such that the geometry remains the same throughout the wave period. Linear hydrodynamic theory is used to calculate the upper and lower bounds for the time-averaged absorbed power and surge foundation loads while assuming that the WEC motion remains sinusoidal. Previous work using pseudospectral techniques to solve the optimal control problem focused solely on maximizing absorbed energy. This work extends the optimal control problem to include a measure of the surge foundation force in the optimization. The objective function includes two competing terms that force the optimizer to maximize power capture while minimizing structural loads. A penalty weight was included with the surge foundation force that allows control of the optimizer performance based on whether emphasis should be placed on power absorption or load shedding. Results from pseudo-spectral optimal control indicate that a unit reduction in time-averaged power can be accompanied by a greater reduction in surge-foundation force.
\end{abstract}

(C) 2016 Published by Elsevier Ltd.

Keywords: oscillating surge wave energy converter, variable structures, load shedding, convex optimization, psuedo-spectral control

\section{Nomenclature}

$\begin{array}{ll}\text { Symbol } & \text { Description } \\ \varphi & \text { Flap pitch angle } \\ \rho_{m} & \text { Structural mass density } \\ h & \text { Water depth } \\ H & \text { OSWEC height } \\ t & \text { OSWEC thickness } \\ w & \text { OSWEC width }\end{array}$

\footnotetext{
${ }^{*}$ Corresponding author

Email addresses: Nathan.Tom@nrel.gov (N. M. Tom ), Yi-Hsiang.Yu@nrel.gov (Y. H. Yu), Alan.Wright@nrel.gov (A. D. Wright), Michael.Lawson@nrel.gov (M. J. Lawson)
} 
Flap width

OSWEC displaced volume

Flap minor axis

Flap major axis

Side support width

Pitch mass moment of inertia

Pitch angular acceleration

Time

Pitch wave-exciting toque

Wave radiation torque due to pitch motion

Hydrostatic restoring torque

Mechanical torque applied by the PTO device

Mass density of the working fluid

WEC displaced volume in calm water

Radial distance from the origin to the center of buoyancy

Mass of the wave energy converter

Radial distance from the origin to the center of gravity

Gravitational acceleration

time-varying pitch angular displacement

$\zeta_{5}$

$C_{55}$

$\mu_{55}$

$K_{r 55}$

Pitch hydrostatic restoring coefficient

Pitch added moment of inertia

$\dot{\zeta}_{5}$

Pitch radiation impulse response function

$\lambda_{55} \quad$ Pitch wave radiation damping

$K_{e 5} \quad$ Pitch wave-excitation torque kernel

$\eta$

$X_{1}$

$\phi_{1}$

$X_{3}$

$\phi_{3}$

$X_{5}$

$\phi_{5}$

$\mathfrak{R}$

$\mathfrak{J}$

$\phi_{I}$

$h$

A

$k$

$\xi_{5}$

$C_{g}$

$B_{g}$

$P_{T}$

$T$

$P_{R}$

$P$

Time-varying incident wave elevation

Frequency-dependent complex surge wave-exciting force coefficient

Frequency-dependent phase of the surge wave-exciting force coefficient

Frequency-dependent complex heave wave-exciting force coefficient

Frequency-dependent phase of the heave wave-exciting force coefficient

Frequency-dependent complex pitch wave-exciting torque coefficient

Frequency-dependent phase of the pitch wave-exciting torque coefficient

Real component

Imaginary component

Incident wave potential

Water depth

Wave amplitude

Wave number

Complex amplitude of the pitch angular displacement

Power-take-off linear restoring coefficient

Power-take-off linear damping coefficient

Time-averaged absorbed power

Wave Period

Time-averaged reactive power

Time-varying instantaneous power

$P_{w} \quad$ Wave time-averaged power per-unit width

$V_{g} \quad$ Wave group velocity

$h \quad$ Water depth

$C_{w} \quad$ Nondimensional capture width: traditional capture width divided by OSWEC width

$C_{r} \quad$ Nondimensional capture width for reactive power

$w \quad$ Width of the OSWEC

$H(x) \quad$ Heaviside step function 


\begin{tabular}{|c|c|}
\hline$\delta$ & Ratio between the constrained-to-optimal pitch angular velocity \\
\hline$X_{r 1}$ & Complex surge reaction force per wave amplitude \\
\hline$X_{r 3}$ & Complex heave reaction force per wave amplitude \\
\hline$\mu_{15}$ & Frequency-dependent surge-pitch radiation added mass \\
\hline$\lambda_{15}$ & Frequency-dependent surge-pitch radiation wave damping \\
\hline$f_{m}$ & Static heave reaction force \\
\hline$f_{r 1}$ & Time-varying surge reaction force \\
\hline$\alpha_{m}$ & Complex amplitude of the PTO torque to eliminate the surge foundation force \\
\hline$E$ & Absorbed energy \\
\hline$N$ & Number of terms in the Fourier series \\
\hline$\varsigma^{c}, \varsigma^{s}$ & Pitch angular displacement cosine and sine Fourier coefficients \\
\hline$\hat{\varsigma}$ & Vector of pitch angular displacement Fourier coefficients \\
\hline$\psi^{c}, \psi^{s}$ & Pitch angular velocity cosine and sine Fourier coefficients \\
\hline$\hat{\psi}$ & Vector of pitch angular velocity Fourier coefficients \\
\hline$\tau^{c}, \tau^{s}$ & Power-take-off torque cosine and sine Fourier coefficients \\
\hline$\hat{\tau}$ & Vector of power-take-off torque Fourier coefficients \\
\hline$\sigma_{0}$ & Fundamental angular frequency \\
\hline$\delta_{i j}$ & Kronecker delta \\
\hline$\theta$ & Cosine and sine time Fourier coefficients \\
\hline$\Phi(t)$ & Vector of time Fourier terms that form the orthogonal basis \\
\hline$\Gamma$ & Time-derivative matrix \\
\hline$G_{55}$ & Pitch wave-radiation convolution integral matrix \\
\hline$\hat{e}_{5}$ & Pitch wave-exciting torque Fourier coefficients \\
\hline$M_{55}$ & Pitch equation of motion matrix \\
\hline$\hat{f}_{r 1}$ & Surge-foundation force Fourier coefficients \\
\hline$\hat{e}_{1}$ & Surge wave-exciting force Fourier coefficients \\
\hline$G_{15}$ & Surge-pitch wave-radiation convolution integral matrix \\
\hline$\gamma$ & Surge-foundation force penalty weight \\
\hline$P_{a b s}$ & Time-averaged absorbed power \\
\hline$C_{R}$ & Ratio of power from optimal control to maximum absorption under motion constraints \\
\hline$F_{r}$ & Ratio of $\left|X_{r 1}\right|$ from optimal control to maximum power absorption under motion constraints \\
\hline
\end{tabular}

Subscript
$p$
$m c$
$z$
$n$

\section{Description}

Passive absorption from setting $C_{g}=0$ and selecting $B_{g} \geq 0$ that maximizes power

Active absorption from selecting $C_{g}$ and $B_{g} \geq 0$ that maximizes power under motion constraints Pitch motion and PTO torque profiles required to eliminate the surge-foundation force Natural (unforced) pitch motion when setting $C_{g}=B_{g}=0$

\section{Introduction}

The success of future wave energy converter (WEC) technologies will require the development of advanced control methods and/or structures that adapt device performance to maximize energy generation in operational conditions while shedding hydrodynamic loads in extreme sea states to reduce the structural mass and overall cost (Musial et al., 2013). In an attempt to address some of these issues, researchers at the National Renewable Energy Laboratory have been developing a novel WEC concept that combines an oscillating surge wave energy converter (OSWEC) with variable geometry (Tom et al., 2016a, 2016b). The design of active control surfaces is expected to assist in tuning the hydrodynamic properties of the device to maximize power absorption in moderate wave climates while shedding loads in larger seas to increase the operational range. The concept of controllable airfoils applied to wave energy conversion has recently been pursued in Atargis Energy Corporation's cycloidal device (Siegel et al., 2011). The idea for largescale geometric changes has been considered in the design of Weptos (Pecher et al., 2012), though the focus has been on its survival mode. The WEC concept used in this paper is more similar to a pitching device with a rotatable flap (Kurniawan and Moan, 2012); however, increasing the number of adjustable surfaces allows for greater refinement in 
the hydrodynamic properties. The development of nearshore OSWECs in recent years has been led by Aquamarine Power's Oyster (Whittaker and Folley, 2012), AW-Energy Oy's Waveroller (Lucas et al., 2012), and Resolute Marine Energy's Surge WEC (Ramudu, 2011). In addition, Langlee Wave Power (Pecher et al., 2010) and PolyGen Ltd are currently developing floating, multiflap OSWECs for deepwater deployment. However, these designs consist of a fixed geometrical body which generally do not operate as a resonant device (Gomes et al., 2015) and instead rely on control of the power-take-off (PTO) system to further optimize power capture.

The control of ocean energy harvesting devices has garnered significant attention in the marine engineering community and is considered necessary for an open-ocean deployment to be successful and economical. Samples of the types of control methodologies that have previously been investigated are complex conjugate (Falnes, 2002), latching (Babarit and Clément, 2006), declutching (Babarit et al., 2009), and inertial tuning (Kurniawan and Moan, 2012 and Flocard and Finnigan, 2012). Applying state-constrained optimization (Eidsmoen, 1996; Hals et al., 2011) to WEC control has gained significant traction in recent times as it provides the ability to include nonlinear constraints. This optimization has been pursued using calculus of variations (Eidsmoen, 1996), model predictive control (Cretel et al., 2011; Abraham and Kerrigan, 2013; Li and Belmont, 2014), and pseudo-spectral methods (Bacelli and Ringwood, 2011 and Herber and Allison, 2013). However, these control strategies focus primarily on maximizing the time-averaged absorbed power without weighing considerations on the corresponding peak forces, torques, and fatigue damage accumulation (Zurkinden et al., 2013). It can be expected that as the controller works to maximize the absorbed mechanical energy, the growth rate in structural loads may exceed the growth in time-averaged power (TAP). This implies that a per-unit gain in TAP may require a greater per-unit gain in structural loading and lead to a power-to-load ratio that peaks and then rapidly decreases. To address this concern, this work incorporates the surge foundation force in the objective function of the controller routine. The controller must now balance two opposing contributions to obtain the largest gain in time-averaged absorbed power with the smallest loads in the foundation of the WEC structure.

This paper begins with a review of the device concept and presentation of the hydrodynamic coefficients for each device geometry considered in this analysis. Next, modeling the OSWEC in the time-domain is reviewed to provide the preliminaries for extension into its spectral representation. Upper and lower bounds on the TAP and surgefoundation force are calculated while assuming that the WEC motion was constrained but sinusoidal. The upper bound was calculated assuming an optimum phase between the pitch wave-exciting torque and pitch angular velocity, and the lower bound assumes that the PTO system consists only of a linear resistive damper, and in both cases the PTO torque coefficients are constant and continuous throughout the wave cycle. The theory of pseduo-spectral control is reviewed, followed by the incorporation of the surge-foundation load in the objective function. A penalty weight is placed on this contribution from the surge-foundation force in an effort to tune the optimal solution to emphasize either power absorption or load shedding. The effect of the penalty weight on optimizer performance was observed by varying its magnitude over a range of wave periods and device geometries. The time history of WEC motion and PTO control torque are presented to illustrate how the increase in TAP can exceed the increase in surge-foundation force as a result of shifting the phase difference between the two.

\section{Device Description and Hydrodynamic Modeling}

As described in previous studies (Tom et al., 2016a, 2016b), the main body of the OSWEC has been replaced with a set of identical flaps that may rotate about its center axis; see Figure 1. The flaps will be allowed to pitch about their center of rotation with the flap pitch angle, $\varphi$, measured positive clockwise from the radial axis of the body; see Figure 2. The flaps were modeled as ellipses to provide a streamlined shape when the flaps are rotated parallel to the direction of wave propagation, $\varphi=\pi / 2$, in an attempt to minimize viscous loses. The geometric dimensions used in this study can be found in Table 2. The structural mass will be evenly distributed, and as such the pitch mass moment of inertia, $I_{55}$, and the linear hydrostatic restoring-torque coefficient, $C_{55}$, will remain constant regardless of flap rotation. For this study the structural mass density, $\rho_{m}$, was set to half the fluid density, $\rho$.

The hydrodynamic coefficients were obtained from WAMIT version 7.0 (WAMIT, 2013) at a spacing of $0.01 \mathrm{rad} / \mathrm{s}$ for wave frequencies between $0 \mathrm{rad} / \mathrm{s}-7.5 \mathrm{rad} / \mathrm{s}$. The flaps were placed in either the closed or fully open configuration corresponding to $\varphi=0$ and $\varphi=\pi / 2$, respectively. The flaps may be controlled independently, but will only be allowed to open in descending order starting with the top flap, labeled as flap 1, which is closest to the free surface. Therefore, flap 3 can only be opened after both flap 1 and flap 2 are already open. This prevents configurations where there may 


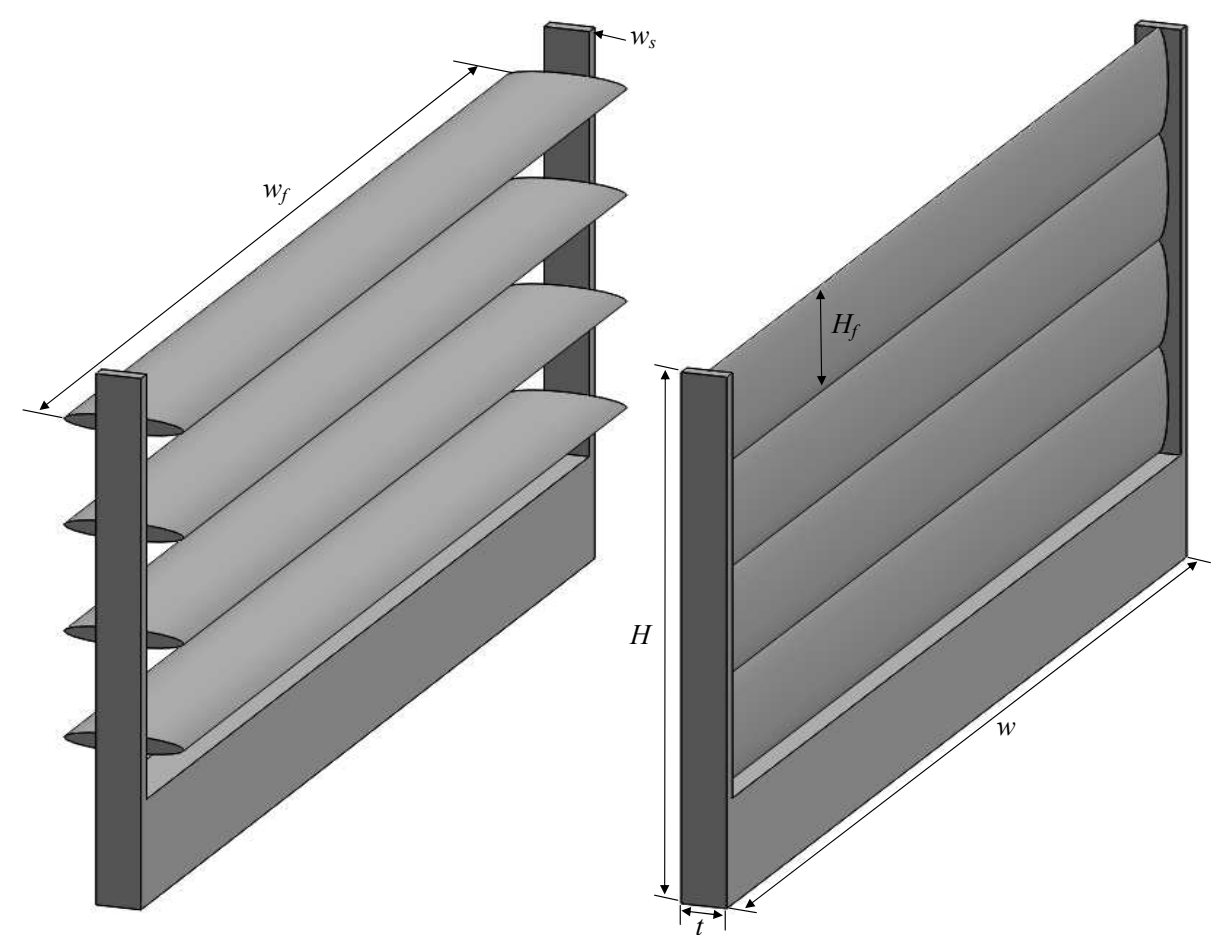

Figure 1. SolidWorks rendering of the OSWEC. Perspective view of fully open configuration (4 flap geometry; left) and perspective view of closed configuration (0 flap geometry; right).

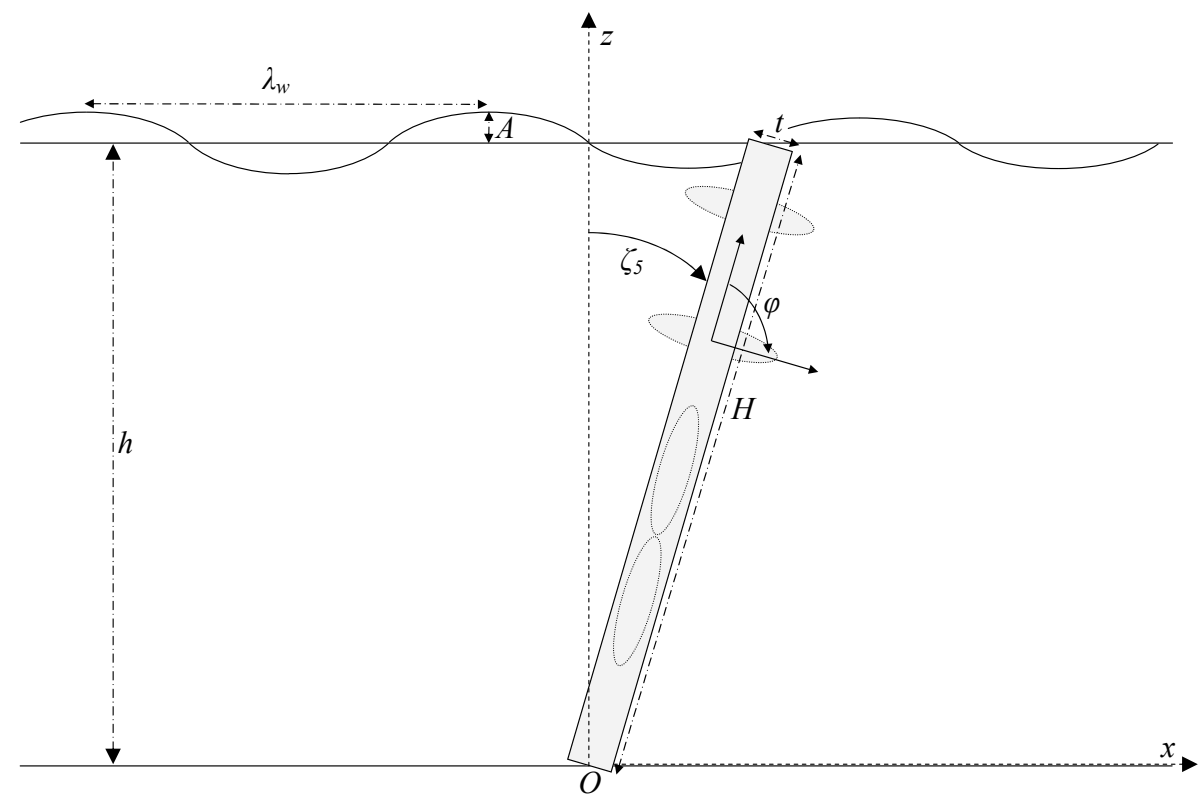

Figure 2. Coordinate system for the device under investigation. Schematic depicts the 2 flap geometry. 
Table 2. Geometric parameters for hydrodynamic modeling.

\begin{tabular}{|c|c|c|c|c|c|}
\hline Water Depth & $h$ & $10 \mathrm{~m}$ & Flap Minor Axis & $t_{f}$ & $1 / 3 \mathrm{~m}$ \\
\hline OSWEC Height & $H$ & $10 \mathrm{~m}$ & Flap Major Axis & $H_{f}$ & $2 \mathrm{~m}$ \\
\hline OSWEC Thickness & $t$ & $3 / 4 \mathrm{~m}$ & Side Support Width & $w_{s}$ & $1 / 4 \mathrm{~m}$ \\
\hline OSWEC Width & $w$ & $20 \mathrm{~m}$ & Center of Gravity & $r_{g}$ & $3.97 \mathrm{~m}$ \\
\hline Flap Width & $w_{f}$ & $19.5 \mathrm{~m}$ & Moment of Inertia & $I_{55}$ & $923.4 \mathrm{~kg} \cdot \mathrm{m}^{2}$ \\
\hline OSWEC Displaced Volume & $\forall$ & $72 \mathrm{~m}^{3}$ & OSWEC Mass & $m$ & $36 \mathrm{t}$ \\
\hline
\end{tabular}

be a closed flap between two open flaps. Results for the closed configuration, no open flaps, are not presented here because it has been previously researched (Bacelli and Ringwood, 2014 and Gomes et al., 2015) and is not required to demonstrate the control benefits from having adjustable structural surfaces. The hydrodynamic coefficients for each geometric configuration can be found in Figure 3 .

\section{Time-Domain Pitch Equation of Motion}

The one-degree-of-freedom time-domain pitch equation of motion is given by:

$$
I_{55} \ddot{\zeta}_{5}(t)=\tau_{e 5}(t)+\tau_{r 55}(t)+\tau_{h}(t)+\tau_{m}(t),
$$

where $t$ is time, $I_{55}$ is the pitch mass moment of inertia, $\ddot{\zeta}_{5}$ is the pitch angular acceleration, $\tau_{e 5}$ is the wave-exciting pitch torque caused by the incident waves, $\tau_{r 55}$ is the wave radiation torque due to pitch motion, $\tau_{h}$ is the hydrostatic restoring torque, and $\tau_{m}$ is the mechanical torque applied by the PTO system.

The OSWEC hydrostatic restoring moment is given by:

$$
\tau_{h}(t)=-\underbrace{\left(\rho \forall r_{b}-m r_{g}\right) g}_{C_{55}} \sin \zeta_{5}(t),
$$

where $\rho$ is the fluid density, $\forall$ is the WEC displaced volume in calm water, $r_{b}$ is the radial distance from the origin to the center of buoyancy, $m$ is the WEC mass, $r_{g}$ is the radial distance from the origin to the center of gravity, $g$ is the gravitational acceleration, and $\zeta_{5}$ is the time-varying pitch angular displacement. Equation (2) can be linearized by assuming small rotational motion, $\zeta_{5} \ll 1$, which allows for the following approximation to be made: $\sin \zeta_{5} \approx \zeta_{5}$.

The linear hydrodynamic wave radiation moment will be represented in the time domain using the Cummins equation (Cummins, 1962) and is written as follows:

$$
\tau_{r 55}(t)=-\mu_{55}(\infty) \ddot{\zeta}_{5}(t)-\int_{-\infty}^{t} K_{r 55}(t-\tau) \dot{\zeta}_{5}(\tau)
$$

where $\mu_{55}(\infty)$ is the pitch added moment of inertia at infinite frequency, and $K_{r 55}$ is the pitch radiation impulse response function, also known as the memory function because it represents the wave radiation memory effect due to past WEC motions. The relations between the time- and frequency-domain radiation coefficients were derived in Ogilvie (1964):

$$
\begin{aligned}
\lambda_{55}(\sigma) & =\int_{0}^{\infty} K_{r 55}(t) \cos \sigma t d t \Rightarrow K_{r 55}(t)=\frac{2}{\pi} \int_{0}^{\infty} \lambda_{55}(\sigma) \cos (\sigma t) d \sigma, \\
\sigma\left[\mu_{55}(\infty)-\mu_{55}(\sigma)\right] & =\int_{0}^{\infty} K_{r 55}(t) \sin \sigma t d t \Rightarrow K_{r 55}(t)=-\frac{2}{\pi} \int_{0}^{\infty} \sigma\left[\mu_{55}(\sigma)-\mu_{55}(\infty)\right] \sin (\sigma t) d \sigma,
\end{aligned}
$$

where $\sigma$ is the wave angular frequency. The variables $\mu_{55}$ and $\lambda_{55}$ are the frequency-dependent hydrodynamic radiation coefficients commonly known as the pitch added moment of inertia and wave damping. 


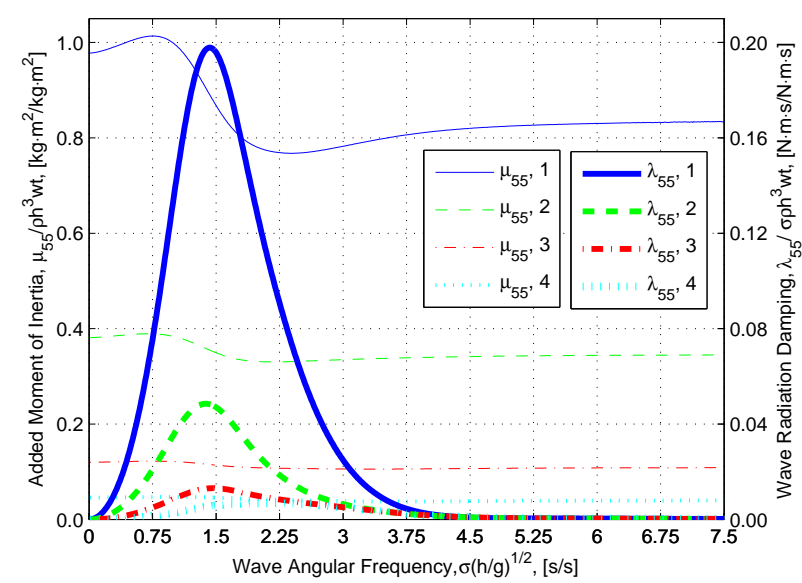

(a) Pitch wave radiation damping, $\lambda_{55}$, and added moment of inertia, $\mu_{55}$.

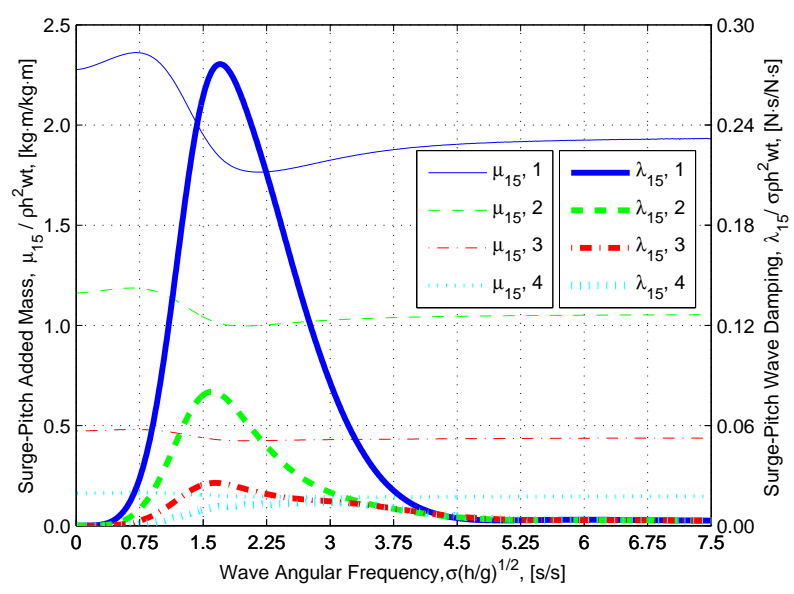

(c) Surge-pitch wave radiation damping, $\lambda_{15}$, and added mass, $\mu_{15}$.

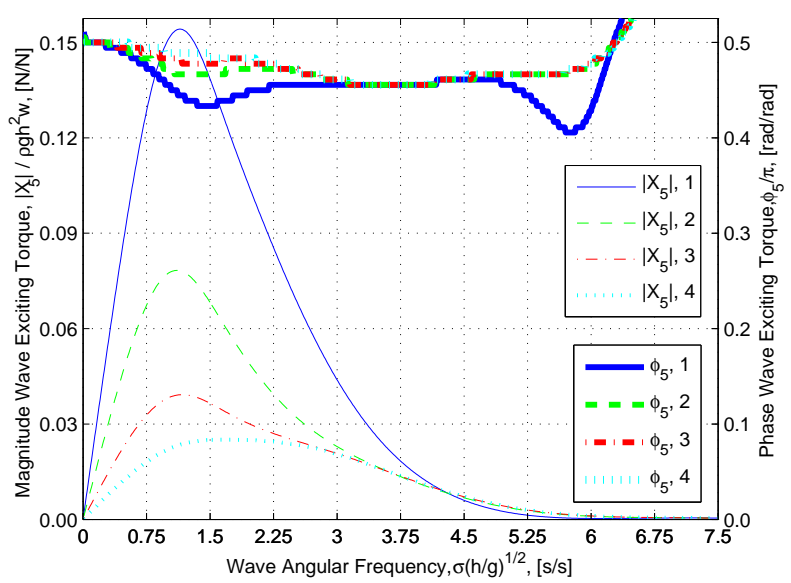

(b) Pitch wave-exciting torque magnitude, $\left|X_{5}\right|$, and phase, $\phi_{5}$.

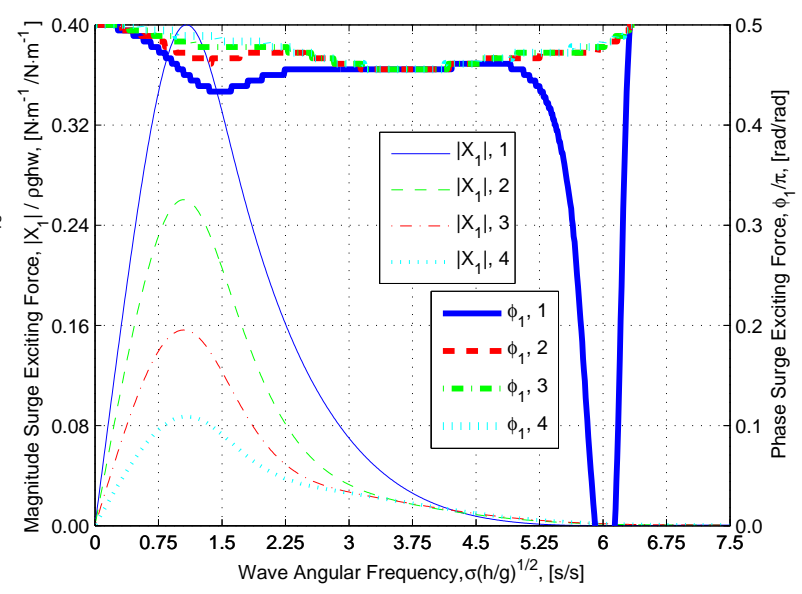

(d) Surge wave-exciting force magnitude, $\left|X_{1}\right|$, and phase, $\phi_{1}$.

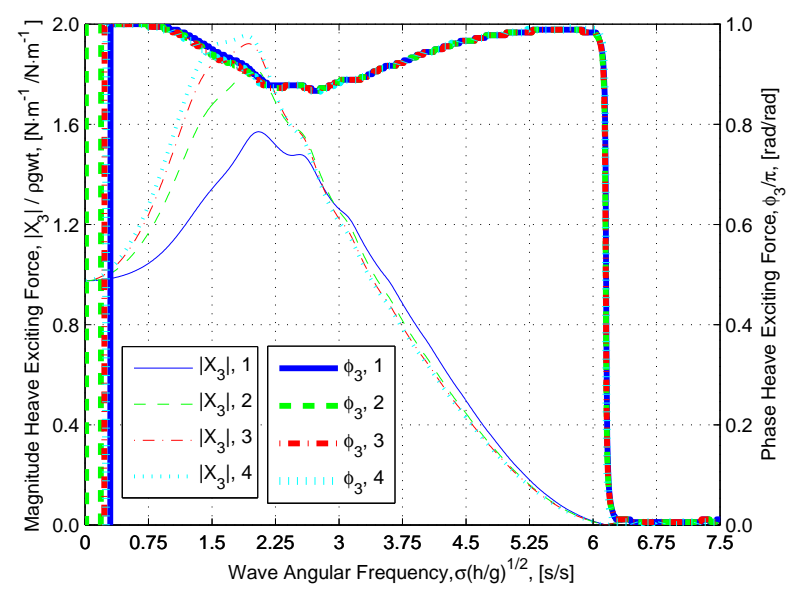

(e) Heave wave-exciting force magnitude, $\left|X_{3}\right|$, and phase, $\phi_{3}$.

Figure 3. Hydrodynamic radiation and wave-excitation coefficients for each flap configuration. The numbers in the legend correspond to the one, two, three, and four flap configurations. 
The wave-exciting pitch torque can be written in the time domain as follows:

$$
\tau_{e 5}(t)=\int_{-\infty}^{\infty} K_{e 5}(t-\tau) \eta(\tau) d \tau
$$

where $K_{e 5}$ is the pitch wave-excitation torque kernal which is noncausal and $\eta$ is the wave elevation. The relationship between the time- and frequency-domain excitation coefficients is given by:

$$
X_{5}(\sigma)=\int_{-\infty}^{\infty} K_{e 5}(t) e^{-i \sigma t} d t \Rightarrow K_{e 5}(t)=\frac{1}{\pi} \int_{0}^{\infty}\left[\mathfrak{R}\left\{X_{5}(\sigma)\right\} \cos (\sigma t)-\mathfrak{J}\left\{X_{5}(\sigma)\right\} \sin (\sigma t)\right] d \sigma,
$$

where $X_{5}$ is the frequency-dependent complex pitch wave-exciting torque coefficient, $\mathfrak{R}$ is the real component, and $\mathfrak{J}$ is the imaginary component.

The final one-degree-of-freedom pitch equation of motion can now be written as:

$$
\left(I_{55}+\mu_{55}(\infty)\right) \ddot{\zeta}_{5}(t)=-C_{55} \zeta_{5}(t)-\int_{-\infty}^{t} K_{r 55}(t-\tau) \dot{\zeta}_{5}(\tau) d \tau+\int_{-\infty}^{\infty} K_{e 5}(t-\tau) \eta(\tau) d \tau+\tau_{m}(t)
$$

\subsection{Regular Wave Analysis \\ 3.1.1. Frequency-Domain Pitch Equation of Motion}

It is common practice to begin analysis under regular wave excitation where the incident wave elevation is described by:

$$
\eta(x, t)=\mathfrak{R}\left\{-\left.\frac{1}{g} \frac{\partial \phi_{I}}{\partial t}\right|_{z=h}\right\}=\mathfrak{R}\left\{A e^{i(\sigma t-k x)}\right\}=A \cos (\sigma t-k x),
$$

where $\eta$ is the wave elevation, $\phi_{I}$ is the incident wave potential, $A$ is the wave amplitude, $k$ is the wave number, and $i=\sqrt{-1}$ is the imaginary unit. The time-harmonic pitch angular response is then given by:

$$
\zeta_{5}(t)=\mathfrak{R}\left\{\xi_{5} e^{i \sigma t}\right\}, \dot{\zeta}_{5}(t)=\mathfrak{R}\left\{i \sigma \xi_{5} e^{i \sigma t}\right\}, \ddot{\zeta}_{5}(t)=\mathfrak{R}\left\{-\sigma^{2} \xi_{5} e^{i \sigma t}\right\}
$$

where $\xi_{5}$ is the complex amplitude of the pitch angular displacement.

Under regular wave excitation, the radiation convolution integral can be simplified when using a change of variables, $\alpha=t-\tau$, as follows:

$$
\tau_{r 55 c}(t)=-\mathfrak{R}\left\{i \sigma \xi_{5} e^{i \sigma t} \int_{0}^{\infty} K_{r 55}(\alpha) e^{-i \sigma \alpha} d \alpha\right\}=\mathfrak{R}\left\{i \sigma \xi_{5} e^{i \sigma t} \int_{0}^{\infty}\left[K_{r 55}(\alpha) \cos \sigma \alpha-i K_{r 55}(\alpha) \sin \sigma \alpha\right] d \alpha\right\},
$$

where Eqns. (4) \& (5) can be used to further simplify the expression:

$$
\tau_{r 55 c}(t)=-\mathfrak{R}\left\{\xi_{5} e^{i \sigma t}\left[\sigma^{2}\left(\mu_{55}(\infty)-\mu_{55}(\sigma)\right)+i \sigma \lambda_{55}\right]\right\} .
$$

The wave-excitation convolution integral can be simplified following the same procedure outlined for the radiation convolution integral, which provides:

$$
f_{e 5}(t)=\mathfrak{R}\left\{A e^{i \sigma t} \int_{-\infty}^{\infty} K_{e 5}(\alpha) e^{-i \sigma \alpha} d \alpha\right\}=\mathfrak{R}\left\{A X_{5}(\sigma) e^{i \sigma t}\right\},
$$

where Eqn. (7) was used.

For the time being, the mechanical torque from the PTO system will be described by the following:

$$
\tau_{m}(t)=-C_{g} \zeta_{5}(t)-B_{g} \dot{\zeta}_{5}(t)=-\mathfrak{R}\left\{\left(C_{g}+i \sigma B_{g}\right) \xi_{5} e^{i \sigma t}\right\},
$$

where $C_{g}$ is the PTO linear-restoring coefficient, $B_{g}$ is the PTO linear-damping coefficient The frequency-domain expressions can be inserted into Eqn. (8), leading to the pitch-displacement response amplitude operator:

$$
\frac{\xi_{5}}{A}=\frac{X_{5}}{\left[C_{55}+C_{g}-\sigma^{2}\left(I_{55}+\mu_{55}\right)\right]+i \sigma\left[\lambda_{55}+B_{g}\right]},
$$




\subsection{PTO Absorbed Power}

The TAP absorbed by the PTO is calculated as follows:

$$
\frac{P_{T}}{A^{2}}=\frac{1}{T A^{2}} \int_{0}^{T}-\tau_{m}(t) \dot{\zeta}_{5}(t) d t=\frac{1}{T A^{2}} \int_{0}^{T}\left[C_{g} \zeta_{5}(t) \dot{\zeta}_{5}(t)+B_{g} \dot{\zeta}_{5}^{2}(t)\right] d t=\frac{1}{2} B_{g} \sigma^{2}\left|\frac{\xi_{5}}{A}\right|^{2},
$$

where $T$ is the wave period. The pitch angular displacement and angular acceleration are out of phase with the angular velocity, resulting in a time-averaged value of zero. Equation (15) can be inserted into Eqn. (16), allowing for the optimal PTO damping at each wave frequency to be calculated. The optimal unconstrained time-averaged absorbed power and PTO damping for each wave frequency is given by:

$$
\begin{aligned}
& \frac{P_{T}}{A^{2}}=\frac{1}{4} \frac{\left|X_{5}\right|^{2}}{\lambda_{55}} \frac{1}{1+\sqrt{1+\left(\frac{C_{55}+C_{g}-\sigma^{2}\left(I_{55}+\mu_{55}\right)}{\sigma \lambda_{55}}\right)^{2}}}, \\
& B_{g}=\lambda_{55} \sqrt{1+\left(\frac{C_{55}+C_{g}-\sigma^{2}\left(I_{55}+\mu_{55}\right)}{\sigma \lambda_{55}}\right)^{2}},
\end{aligned}
$$

where at resonance $B_{g}=\lambda_{55}$, leading to the maximum time-averaged absorbed power (Evans, 1976). Because these expressions do not consider motion constraints, it may be necessary to increase the PTO damping to remain under a given limit. The required PTO damping is given by:

$$
B_{g}=\left\{\left(\frac{A\left|X_{5}\right|}{\sigma\left|\xi_{5}\right|_{\max }}\right)^{2}-\left[\frac{C_{55}+C_{g}}{\sigma}-\sigma\left(I_{55}+\mu_{55}\right)\right]^{2}\right\}^{1 / 2}-\lambda_{55},
$$

where $\left|\xi_{5}\right|_{\max }$ is the maximum amplitude of pitch displacement (Hals et al., 2002). The time averaged reactive power, defined as the power that the PTO returns to the oscillating body, is calculated from:

$$
P_{R}=\frac{1}{T} \int_{0}^{T} \min [P(t), 0] d t
$$

where $P(t)$ is the PTO instantaneous power. To provide a measure of the efficiency for a given device, the TAP contained within a propagating wave must be known. The time-averaged wave power per-unit width, $P_{w}$, can be obtained from:

$$
P_{w}=\frac{1}{2} \rho g A^{2} V_{g}=\frac{1}{4} \rho g A^{2} \sqrt{\frac{g}{k} \tanh k h}\left[1+\frac{2 k h}{\sinh k h}\right] .
$$

where $V_{g}$ is the wave group velocity, and $h$ is the water depth. The nondimensional capture width in this work will be defined as:

$$
C_{w}=\frac{P_{T}}{w P_{w}}
$$

where $w$ is the width of the OSWEC.

\subsubsection{Power Quality}

Falnes (2002a) and Hals et al. (2002) show that under regular wave-excitation, the instantaneous power, $P$, passing through the PTO is given by:

$$
P(t)=P_{T}+\frac{1}{2}\left|i \sigma \xi_{5}\right|^{2}|\underbrace{B_{g}+i C_{g}}_{Z}| \cos (2(\sigma t+v)+\vartheta)
$$

where $v$ is the argument of $i \sigma \xi_{5}$ and $\vartheta$ is the argument of $Z$. As seen from Eqn. (23), the instantaneous power fluctuates about a mean given by the TAP with an amplitude given by the complex impedance, $Z$. Often in power 
quality management it is desirable to have the peak-to-average power ratio as close as possible to eliminate the need for advanced signal conditioning. The peak-to-average power ratio, $P A_{ \pm}$, can be calculated from:

$$
P A_{ \pm}=1 \pm \frac{|Z|}{B_{g}}=1 \pm \sqrt{1+\left[\frac{C_{g}}{\sigma B_{g}}\right]^{2}}
$$

When there is no reactive power, $C_{g}=0$, the peak-to-average power ratio is 2 and the instantaneous power oscillates between 0 and $2 P_{T}$. The reactive component is eliminated at the resonance frequency of the isolated floating body and the peak-to-average power ratio is minimized at 2; however, when moving away from the resonant frequency, the peak-to-average power ratio quickly increases resulting in large swings in the bidirectional energy flow. It is possible to lower the peak-to-average power ratio by increasing the PTO damping coefficient at the expense of reduced pitch motion and power absorption, see Eqn. (16).

\subsubsection{Maximum Power under Constrained Motion}

The maximum power absorption under pitch amplitude constraints, while assuming sinusoidal motion, was explored in Evans (1981) which led to the following expression:

$$
\frac{P_{T}}{A^{2}}=\frac{1}{8} \frac{\left|X_{5}\right|^{2}}{\lambda_{55}}\left[1-H(1-\delta)(1-\delta)^{2}\right],
$$

where $H(x)$ is the Heaviside step function, and $\delta$ is the ratio between the constrained-to-optimal pitch angular velocity given by:

$$
\delta=\frac{\sigma\left|\xi_{5}\right|_{\max }}{A} \frac{2 \lambda_{55}}{\left|X_{5}\right|} .
$$

Equation (25) can be expanded to show the trends in time-averaged absorbed power for the ranges of $\delta$ :

$$
P_{T}= \begin{cases}\frac{1}{8} A^{2}\left|X_{5}\right|^{2} / \lambda_{55} & , \delta>1 \\ \frac{1}{2} A\left|X_{5}\right| \sigma\left|\xi_{5}\right|_{\text {max }}-\lambda_{55} \sigma^{2}\left|\xi_{5}\right|_{\text {max }}^{2} & , \delta<1\end{cases}
$$

The capture width, defined as the ratio between the TAP absorbed by the PTO and the incident wave power per-unit width is a metric used to evaluate the absorption efficiency of the device. The incident wave power is proportional to the incident wave amplitude squared, see Eqn. (21). For unconstrained motion, which may also correspond to a very small incident wave amplitude, the capture width will be invariant to the incident wave height; whereas for a strongly constrained motion, which may also correspond to a very large incident wave amplitude, the capture width will be inversely proportional to the incident wave height absorbing a smaller portion of the available wave power.

The associated PTO linear-damping coefficient required to observe the motion constraint is given by:

$$
B_{g}=\lambda_{55}\left[1+\frac{2(1-\delta)}{\delta} H(1-\delta)\right]=\left\{\begin{array}{ll}
\lambda_{55} & , \delta>1 \\
\frac{A\left|X_{5}\right|}{\sigma\left|\xi_{5}\right| \max }-\lambda_{55} & , \delta<1
\end{array},\right.
$$

and the PTO spring coefficient must combine to cancel the dynamic force contribution from the natural-body-hydrostatic restoring coefficient, mass moment of inertia, and hydrodynamic added moment of inertia:

$$
C_{g}=-\left[C_{55}-\sigma^{2}\left(I_{55}+\mu_{55}\right)\right],
$$

which is the basis of complex conjugate control (Falnes, 2002).

\subsection{Foundation Reaction Forces}

The structural foundation must handle the reaction forces needed to fix the WEC to the seabed. If centrifugal forces are neglected and the body remains symmetric about the vertical plane, the foundation forces in the surge, $X_{r 1}$, and heave, $X_{r 3}$, directions are given by:

$$
\begin{gathered}
A\left(X_{r 1}+X_{1}\right)=\left[-\sigma^{2} \mu_{15}+i \sigma \lambda_{15}\right] \xi_{5}, \\
A\left(X_{r 3}+X_{3}\right)+\underbrace{(\rho \forall-m) g}_{f_{m}}=0,
\end{gathered}
$$


where $X_{1}$ and $X_{3}$ are the complex surge and heave wave-exciting force coefficients per unit wave amplitude, $\mu_{15}$ is the surge-pitch added mass, and $\lambda_{15}$ is the surge-pitch wave radiation damping. The static heave reaction force, $f_{m}$, associated with the difference between weight and buoyancy is significant when calculating peak forces, but it is not a dynamic force and cannot be controlled through either the PTO or geometry. The dynamic forces will drive fatigue damage accumulation in the foundation rather than the static mean load. In very small sea states the static heave force will dominate, but as the wave amplitude grows the dynamic component of the foundation forces will have a larger contribution in the magnitude of the foundation forces. The following analysis will not include the static reaction force associated with the difference between weight and buoyancy and focus on the dynamic forces. The above equations can be simplified and written in terms of the surge wave-exciting force and pitch motion:

$$
\begin{gathered}
X_{r 1}=\left[-\sigma^{2} \mu_{15}+i \sigma \lambda_{15}\right] \frac{\xi_{5}}{A}-X_{1}, \\
X_{r 3} \approx-X_{3} .
\end{gathered}
$$

The heave foundation force is not affected by the body motion and only varies with device geometry. The surgefoundation force is affected by the pitch motion of the OSWEC, which can be controlled by the PTO. The time-domain representation of Eqn. (32) is given by:

$$
f_{r 1}(t)=-\int_{-\infty}^{\infty} K_{e 1}(t-\tau) \eta(\tau) d \tau+\mu_{15}(\infty) \ddot{\zeta}_{5}(t)+\int_{-\infty}^{t} K_{r 15}(t-\tau) \dot{\zeta}_{5}(\tau) d \tau
$$

where $K_{e 1}$ is the sure wave-excitation force kernel and $K_{r 15}$ is the surge-pitch radiation impulse response function. The surge-foundation force can theoretically be eliminated if the complex pitch angular displacement amplitude of motion is equal to:

$$
\frac{\xi_{5}}{A}=\frac{X_{1}}{-\sigma^{2} \mu_{15}+i \sigma \lambda_{15}}=\frac{-\mu_{15} \mathfrak{R}\left\{X_{1}\right\}+\frac{\lambda_{15}}{\sigma} \mathfrak{J}\left\{X_{1}\right\}}{\sigma^{2} \mu_{15}^{2}+\lambda_{15}^{2}}+i \frac{-\mu_{15} \mathfrak{J}\left\{X_{1}\right\}-\frac{\lambda_{15}}{\sigma} \mathfrak{R}\left\{X_{1}\right\}}{\sigma^{2} \mu_{15}^{2}+\lambda_{15}^{2}},
$$

which requires the following complex PTO control torque amplitude, $\alpha_{m}$ :

$$
\frac{\alpha_{m}}{A}=\frac{\left[C_{55}-\sigma^{2}\left(I_{55}+\mu_{55}\right)+i \sigma \lambda_{55}\right] X_{1}}{\left[-\sigma^{2} \mu_{15}+i \sigma \lambda_{15}\right]}-X_{5} .
$$

The magnitude and phase of the pitch angular velocity required to eliminate the surge reaction force has been plotted in Fig. 4(a) and Fig. 4(b). These plots show that for this OSWEC design the natural body motion closely follows the pitch dynamics required to eliminate the surge foundation force for wave frequencies above the resonant frequency of each geometry; however, when below the resonance frequency the pitch motion must be amplified which requires an increase the PTO control torque amplitude, see Fig. 4(c). As the WEC begins to absorbed power from the incident waves the change in magnitude and phase of the pitch motion will lead to an increase in the surge foundation force. As both the magnitude and phase of WEC motion contributes to the surge foundation force it may be possible to control the pitch dynamics to obtain a greater rate of growth in power absorption than structural loading.

\subsection{Results from Fixed-PTO Coefficients}

Maximizing the TAP, as described in the previous sections, considers the PTO coefficients to be fixed in time although tuned for a given wave amplitude and angular frequency. Performance bounds can be set for the TAP and surge-foundation force amplitude and have been plotted in Fig. 5(a) and Fig. 5(b). It is expected that time-varying PTO coefficients may help optimize the TAP while reducing structural loads, leading to device performance that sits between the maximum constrained and passive curves. The performance curves in Fig. 5(a) show that as each additional flap is opened, the maximum nondimensional capture width decreases, specifically in the low-frequency regime, where the 1-flap geometry peaks at just over 1.6. However, the reactive power required to achieve that power absorption can be up to 5 times the TAP output power, see Fig. 5(e). The reduction in peak capture width between the 1-flap and 4-flap geometries is $45 \%$ with a corresponding decrease in the reactive capture width of approximately $75 \%$. 


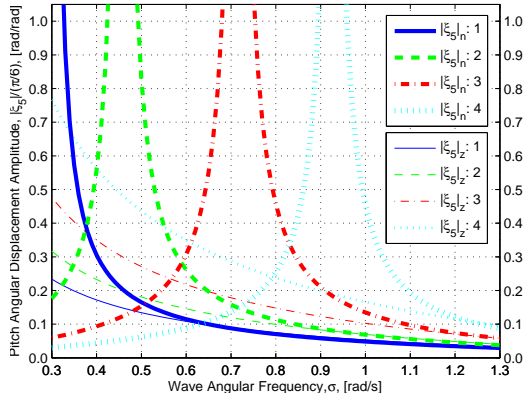

(a) Natural \& Zero Surge-Foundation Force Pitch Displacement Amplitude

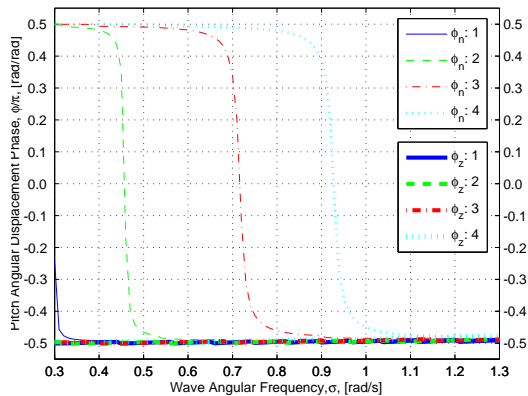

(b) Natural \& Zero Surge-Foundation Force (c) PTO Torque \& Pitch Velocity Amplitude for Pitch Displacement Phase

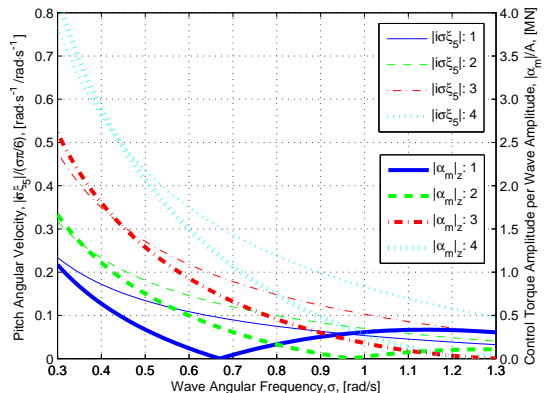

Zero Surge-Foundation Force

Figure 4. The magnitude and phase of the pitch angular displacement, pitch angular velocity, and PTO torque for a wave amplitude of $0.125 \mathrm{~m}$ and varying wave angular frequency. The subscript ${ }_{n}$ denotes the unforced (no PTO $C_{g}=B_{g}=0$ ) motion while the subscript $z_{z}$ denotes the pitch angular motion and PTO torque required for elimination of the surge-foundation force as given by Eqns. (35)-(36). The numbers in the legend correspond to the number of flaps rotated to $\varphi=90$, which starts with the top flap and each additional flap is opened from the top-down.

The maximum surge-foundation force amplitude has dropped by $80 \%$ between the same geometries, see Fig. 5(b), which indicates that a greater reduction in loads can be achieved per unit reduction in TAP. It is of interest to note that as each additional flap is opened, the wave frequency at which the maximum constrained surge-foundation force amplitude is greatest moves closer to the resonant frequency, refer to Fig. 5(d). Further, the surge-foundation force amplitude is considerably smaller than the static reaction force, $f_{m}$, for specific geometric configurations; however, the results plotted are for a small-incident wave amplitude, and with increasing wave height the dynamic force will become comparable to the static. As shown in Fig. 5(c), the PTO control torque amplitude varies significantly with device geometry and incident wave angular frequency. For maximum constrained performance, the required torque amplitude is minimized at the resonance frequency of each geometric configuration. The greatest power capture can be achieved with only the 1-flap geometry, but the required torque amplitude will lead to significant fatigue damage accumulation as it is proportional to the torque amplitude cubed (Tom et. al, 2016b). It is evident from Fig. 5(f), that the peak-to-average power ratio is significantly larger than the increase in time-averaged power when moving $15 \%$ below or above the resonance frequency of each geometry. The authors believe that when the peak in the instantaneous reactive power, $\min (P)$, is equal to the TAP limits, the feasibility of complex conjugate control because issues are likely to arise with the PTO rated power.

\section{Pseudo-spectral Optimal Control}

A more detailed derivation into construction of the pseudo-spectral optimal control problem is given in Bacelli and Ringwood (2015), and a brief review is provided in this section. The optimal control problem consists of obtaining the PTO torque vector, $\tau_{m}$, that maximizes the total absorbed energy, $E$, subject to the equations of motion and additional control and motion constraints. These conditions are generally written as:

$$
\begin{gathered}
\min E=\int_{0}^{T} \dot{\zeta}_{5}(t) \tau_{m}(t) d t, \\
h\left(\zeta_{5}, \dot{\zeta}_{5}, \tau_{m}, t\right)=0, \\
g\left(\zeta_{5}, \dot{\zeta}_{5}, \tau_{m}, t\right) \leq 0 .
\end{gathered}
$$

The discretization of the control problem is completed by approximating the position, velocity, and PTO torque with a linear combination of basis functions. The pitch angular displacement, pitch angular velocity, and control torque are 

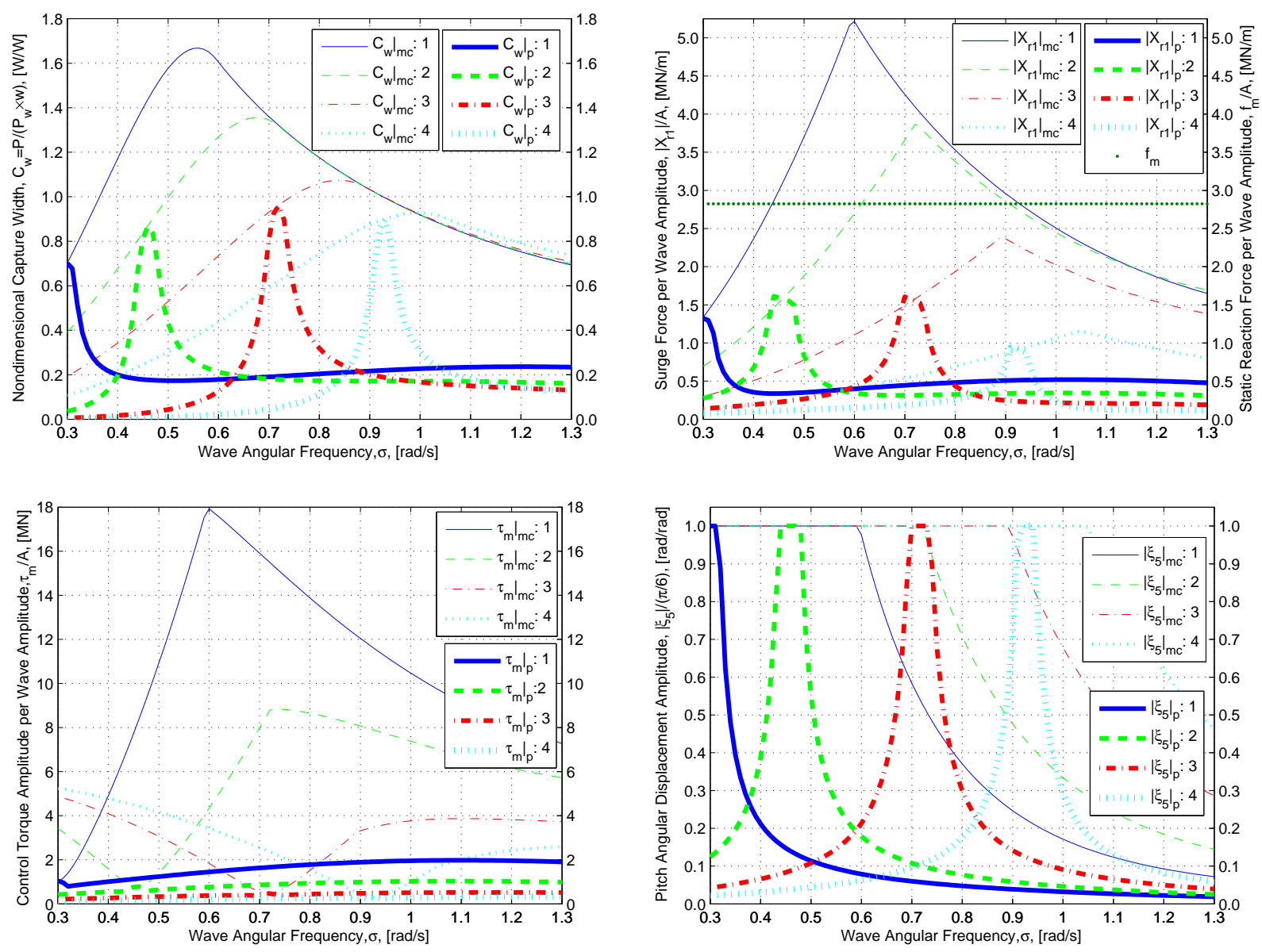

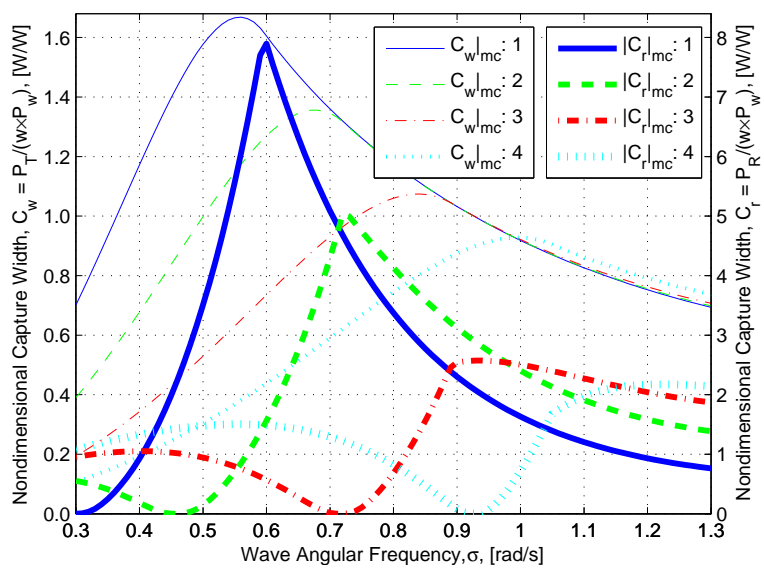

(e) Nondimensional Capture Width and Reactive Capture Width

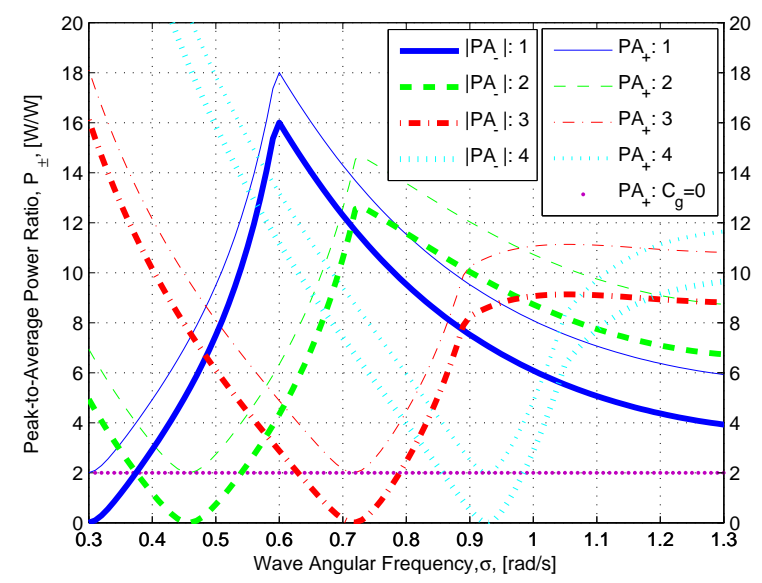

(f) Peak-to-Average Power Ratios

Figure 5. Performance bounds for each geometric configuration with a pitch-displacement constraint of $\pi / 6$ and a wave amplitude of $0.125 \mathrm{~m}$. The subscript ${ }_{p}$ denotes performance for passive power as given by Eqns. (16)-(19) while setting $C_{g}=0$. The subscript ${ }_{m c}$ denotes performance for maximum constrained power as given by Eqns. (25)-(29). The numbers in the legend correspond to the number of flaps rotated to $\varphi=90$, which starts with the top flap and each additional flap is opened from the top-down. 
approximated by zero-mean truncated Fourier series with $N$ terms:

$$
\begin{gathered}
\zeta_{5}(t) \approx \sum_{j=1}^{N / 2} \varsigma_{j}^{c} \cos \left(j \sigma_{0} t\right)+\varsigma_{j}^{s} \sin \left(j \sigma_{0} t\right)=\Phi(t) \hat{\varsigma}, \\
\dot{\zeta}_{5}(t) \approx \sum_{j=1}^{N / 2} \psi_{j}^{c} \cos \left(j \sigma_{0} t\right)+\psi_{j}^{s} \sin \left(j \sigma_{0} t\right)=\Phi(t) \hat{\psi}, \\
\tau_{m}(t) \approx \sum_{j=1}^{N / 2} \tau_{j}^{c} \cos \left(j \sigma_{0} t\right)+\tau_{j}^{s} \sin \left(j \sigma_{0} t\right)=\Phi(t) \hat{\tau},
\end{gathered}
$$

where

$$
\begin{gathered}
\hat{\varsigma}=\left[\varsigma_{1}^{c}, \varsigma_{1}^{s}, \ldots, \varsigma_{\frac{N}{2}}^{c}, \varsigma_{\frac{N}{2}}^{s}\right]^{\top}, \hat{\psi}=\left[\psi_{1}^{c}, \psi_{1}^{s}, \ldots, \psi_{\frac{N}{2}}^{c}, \psi_{\frac{N}{2}}^{s}\right]^{\top}, \hat{\tau}=\left[\tau_{1}^{c}, \tau_{1}^{s}, \ldots, \tau_{\frac{N}{2}}^{c}, \tau_{\frac{N}{2}}^{s}\right]^{\top}, \\
\Phi(t)=\left[\theta_{1}(t), \theta_{2}(t), \ldots, \theta_{N-1}(t), \theta_{N}(t)\right]=\left[\cos \left(\sigma_{0} t\right), \sin \left(\sigma_{0} t\right), \ldots, \cos \left(\frac{N}{2} \sigma_{0} t\right), \sin \left(\frac{N}{2} \sigma_{0} t\right)\right],
\end{gathered}
$$

where $\Phi(t)$ is the vector of time Fourier terms that form the orthogonal basis and the fundamental angular frequency is given by $\sigma_{0}=2 \pi / T$. The vector $\hat{s}$ consists of the pitch angular displacement cosine, $\varsigma_{j}^{c}$, and sine, $\varsigma_{j}^{s}$, Fourier coefficients. The vector $\hat{\psi}$ consists of the pitch angular velocity cosine, $\psi_{j}^{c}$, and sine, $\psi_{j}^{s}$, Fourier coefficients. The final vector $\hat{\tau}$ consists of the PTO torque cosine, $\tau_{j}^{c}$, and sine, $\tau_{j}^{s}$, Fourier coefficients. The absorbed energy, $E$, can now be approximated as:

$$
E=-\int_{0}^{T} \hat{\psi}^{\top} \Phi(t)^{\top} \Phi(t) \hat{\tau} d t=-\frac{T}{2} \hat{\psi}^{\top} \hat{\tau},
$$

where ${ }^{\top}$ denotes transpose. The final form of the absorbed energy is a result of the choice in basis functions:

$$
\left\langle\phi_{i}, \phi_{j}\right\rangle=\int_{0}^{T} \phi_{i}(t) \phi_{j}(t) d t=\frac{T}{2} \delta_{i j},
$$

where $\delta_{i j}$ is the Kronecker delta.

\subsection{Pitch Equations of Motion}

The equality constraints are used to satisfy the system equations of motion. The time derivative of the pitch angular displacement, when using a Fourier series representation, can be written as:

$$
\dot{\zeta}_{5}=\dot{\Phi}(t) \hat{\varsigma}=\Phi(t) \Gamma \hat{\varsigma},
$$

where the time-derivative matrix, $\Gamma \in \mathbb{R}^{N \times N}$, is block diagonal with the following block structure:

$$
\Gamma^{j}=\left[\begin{array}{cc}
0 & j \sigma_{0} \\
-j \sigma_{0} & 0
\end{array}\right], \text { for } j=1,2, \ldots, N / 2 .
$$

The dynamic relationship between pitch angular displacement and angular velocity leads to the following expression:

$$
\Phi(t) \Gamma \hat{\varsigma}=\Phi(t) \hat{\psi} \Rightarrow \hat{\varsigma}=\Gamma^{-1} \hat{\psi},
$$

which allows Eqn. (8) to take the following form:

$$
\left(I_{55}+\mu_{55}(\infty)\right) \Phi(t) \Gamma \hat{\psi}+C_{55} \Phi(t) \Gamma^{-1} \hat{\psi}+\Phi(t)\left(G_{55}-\mu_{55}(\infty) \Gamma\right) \hat{\psi}-\Phi(t) \hat{\tau}-\Phi(t) \hat{e}_{5}=0,
$$

where $G_{55}$ is the wave-radiation convolution integral matrix and $\hat{e}_{5}$ is the Fourier coefficients of the pitch wave-exciting torque. The wave-radiation convolution integral can be decomposed into the above matrix form with the derivation left to Appendix A. The equation can further be simplified as follows:

$$
M_{55} \hat{\psi}=\hat{\tau}+\hat{e}_{5},
$$


where the matrix $M_{55} \in \mathbb{R}^{N \times N}$ is block diagonal with the following structure:

$$
M_{55}^{j}=\left[\begin{array}{cc}
\lambda_{55}\left(j \sigma_{0}\right) & j \sigma_{0}\left(I_{55}+\mu_{55}\left(j \sigma_{0}\right)\right)-C_{55} /\left(j \sigma_{0}\right) \\
-j \sigma_{0}\left(I_{55}+\mu_{55}\left(j \sigma_{0}\right)\right)+C_{55} /\left(j \sigma_{0}\right) & \lambda_{55}\left(j \sigma_{0}\right)
\end{array}\right], \text { for } j=1,2, \ldots, N / 2 .
$$

The pitch angular velocity Fourier coefficients can be determined explicitly from the control-torque and pitch waveexcitation torque Fourier coefficients. This representation allows Eqn. (45) to be written as:

$$
E=-\frac{T}{2}\left[\hat{\tau}^{\top}\left(M_{55}^{-1}\right)^{\top} \hat{\tau}+\hat{e}_{5}^{\top}\left(M_{55}^{-1}\right)^{\top} \hat{\tau}\right],
$$

which is in the form of a traditional quadratic problem. The symmetric component of $M_{55}^{-1}$ is positive definite (Bacelli and Ringwood, 2015), which guarantees that the problem is convex and the solution is globally optimal.

\subsection{Amplitude and Torque Constraints}

Constraints on the PTO torque and pitch-displacement amplitude reflect the physical limits of the system. These limits will be modeled as inequality constraints:

$$
\begin{gathered}
\Phi(t) \hat{\tau} \leq\left. 1_{(N \times 1)} \tau_{m}\right|_{\max }, \\
-\Phi(t) \hat{\tau} \leq-\left.1_{(N \times 1)} \tau_{m}\right|_{\min }, \\
\Phi(t) \Gamma^{-1} M_{55}^{-1} \hat{\tau} \leq\left. 1_{(N \times 1)} \zeta_{5}\right|_{\max }-\Phi(t) \Gamma^{-1} M_{55}^{-1} \hat{e}_{5}, \\
-\Phi(t) \Gamma^{-1} M_{55}^{-1} \hat{\tau} \leq-\left.1_{(N \times 1)} \zeta_{5}\right|_{\min }+\Phi(t) \Gamma^{-1} M_{55}^{-1} \hat{e}_{5} .
\end{gathered}
$$

The constraints are enforced at specific time instants given by $t_{k}=k T /(N+1)$ for $k=1,2, \ldots N$ (Bacelli and Ringwood, 2015). The constrained optimal control problem is now a convex quadratic program subject to linear constraints on the PTO torque and pitch displacement amplitude. The impact of device motion and force constraints have also been discussed in Garcia-Rosa et. al (2015); however, in this work no constraints were placed on the PTO control torque.

\subsection{Surge-Foundation Force}

The majority of the research on optimal control has focused on maximizing the TAP; however, it is well-understood that this will require large PTO torque amplitudes and greater structural loading. It is of interest to include a representation of the associated surge foundation load to the objective function. This will allow the optimizer to find a balance between the TAP and surge-foundation loads based on the desires of the designer. For this to be implemented, Equation (34) will be written in a matrix form, as follows:

$$
\begin{gathered}
\Phi(t) \hat{f}_{r 1}=-\Phi(t) \hat{e}_{1}+\mu_{15}(\infty) \Gamma \hat{\psi}+\Phi(t)\left(G_{15}-\mu_{15}(\infty) \Gamma\right) \hat{\psi}, \\
\hat{f}_{r 1}=-\hat{e}_{1}+G_{15} \hat{\psi}=-\hat{e}_{1}+G_{15} M_{55}^{-1} \hat{\tau}+G_{15} M_{55}^{-1} \hat{e}_{5},
\end{gathered}
$$

where $\hat{e}_{1}$ is the Fourier coefficients of the surge wave-exciting force and where Eqn. (51) has been substituted in the last expression. The block matrix $G_{15}$ has the same form as $G_{55}$ (see Appendix A), except the surge-pitch radiation coefficients are used instead. To maintain the convexity of the quadratic problem, the squared $\ell^{2}$-norm of the surgefoundation force vector was added to the objective function.

$$
\begin{aligned}
\left|f_{r 1}\right|^{2}= & \int_{0}^{T} \hat{f}_{r 1}^{\top} \Phi(t)^{\top} \Phi(t) \hat{f}_{r 1} d t=\frac{T}{2} \hat{f}_{r 1}^{\top} \hat{f}_{r 1} \\
= & \frac{T}{2}\left[\hat{e}_{1}^{\top} \hat{e}_{1}-2 \hat{e}_{1}^{\top} G_{15} M_{55}^{-1} \hat{e}_{5}+\hat{e}_{5}^{\top}\left(M_{55}^{-1}\right)^{\top} G_{15}^{\top} G_{15} M_{55}^{-1} \hat{e}_{5}\right. \\
& \left.+2 \hat{e}_{5}^{\top}\left(M_{55}^{-1}\right)^{\top} G_{15}^{\top} G_{15} M_{55}^{-1} \hat{\tau}-2 \hat{e}_{1}^{\top} G_{15} M_{55}^{-1} \hat{\tau}+\hat{\tau}^{\top}\left(M_{55}^{-1}\right)^{\top} G_{15}^{T} G_{15} M_{55}^{-1} \hat{\tau}\right] .
\end{aligned}
$$

In the final expression for $\left|f_{r 1}\right|^{2}$ there are three constant terms independent of the control torque, which will be left out of the optimization. The final objective function is now given by:

$$
J=-\frac{T}{2}\left[\hat{\tau}^{\top} M_{55}^{-1} \hat{\tau}+\hat{\tau}^{\top} M_{55}^{-1} \hat{e}_{5}+\gamma\left(-2 \hat{e}_{5}^{\top}\left(M_{55}^{-1}\right)^{\top} G_{15}^{\top} G_{15} M_{55}^{-1} \hat{\tau}+2 \hat{e}_{1}^{\top} G_{15} M_{55}^{-1} \hat{\tau}-\hat{\tau}^{\top}\left(M_{55}^{-1}\right)^{\top} G_{15}^{T} G_{15} M_{55}^{-1} \hat{\tau}\right)\right]
$$

where $\gamma$ is a penalty weight that can be used to adapt the optimizer performance. 

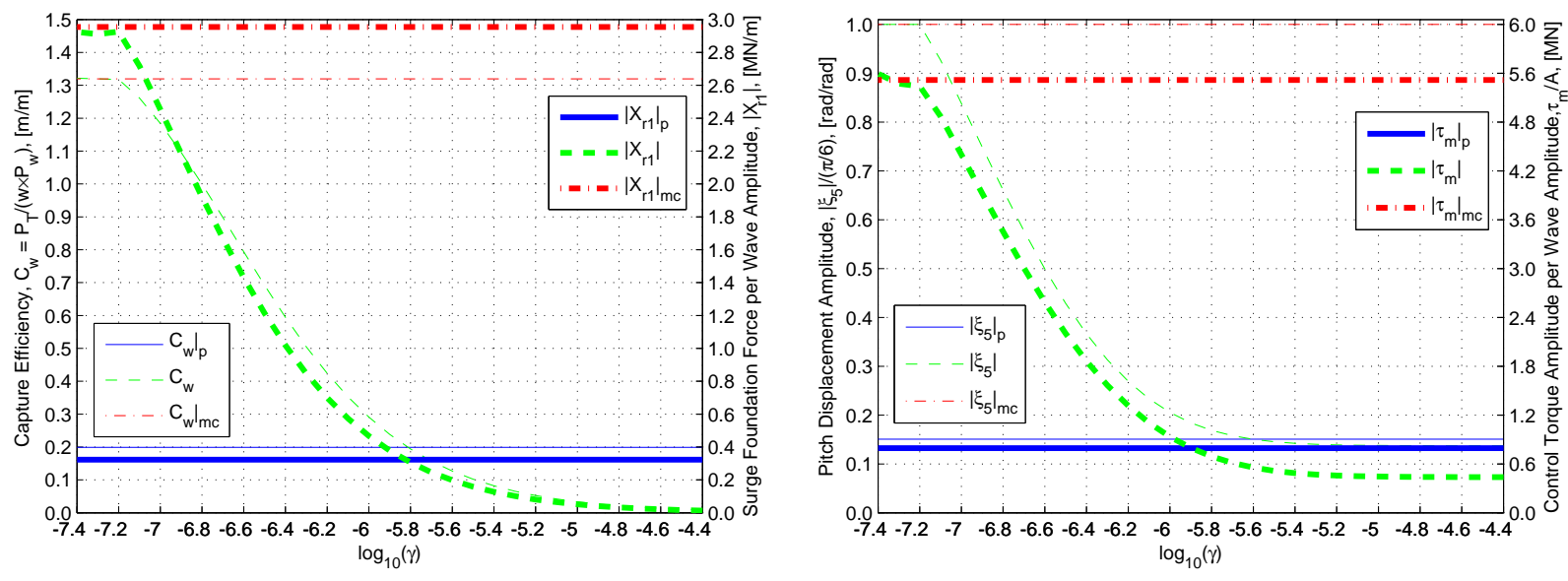

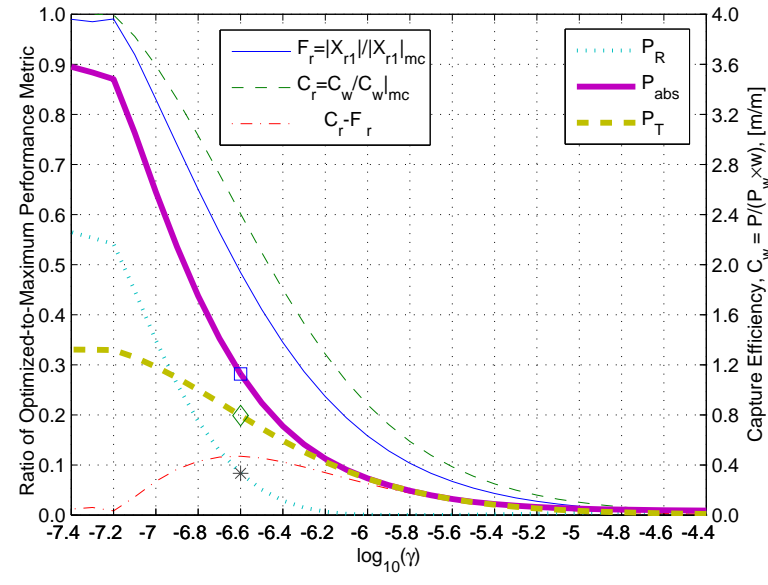

(c) Sensitivity of $C_{r}$ and $F_{r}$ with respect to $\gamma$

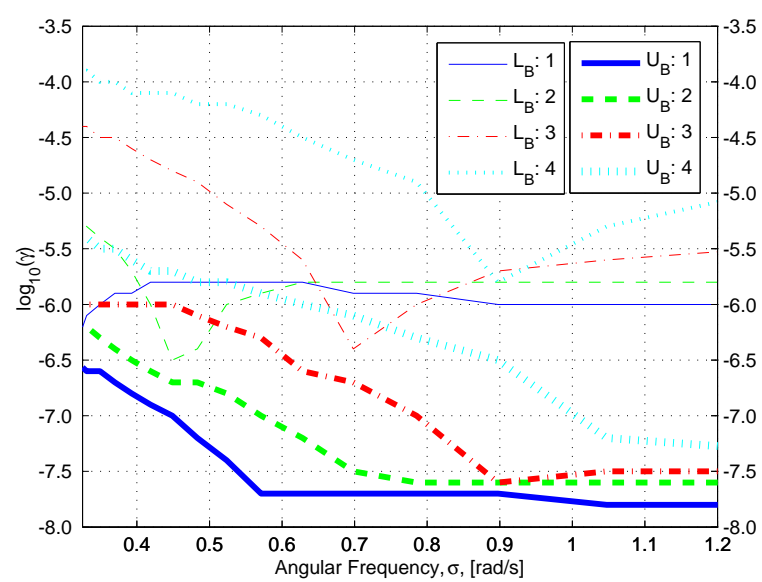

(d) Upper and lower bounds on the penalty weight $\gamma$

Figure 6. Performance sensitivity to force penalty weight. Results are plotted for the 2-flap geometric configuration with a pitch-displacement amplitude limit of $\pi / 6$, a wave amplitude of $0.125 \mathrm{~m}$, and a wave period of $10 \mathrm{~s}$. The subscript $m c$ denotes performance for maximum constrained power as given by Eqns. (25)-(29). The subscript $p$ denotes performance for passive power as given by Eqns. (16)-(19).

\subsubsection{Surge-Foundation Force Constraint}

Rather than weight the contribution from the surge-foundation force in the objective function, it can also be used as an inequality constraint to limit its amplitude:

$$
\begin{gathered}
\Phi(t) G_{15} M_{55}^{-1} \hat{\tau} \leq\left. 1_{(N \times 1)} f_{r 1}\right|_{\max }+\Phi(t) \hat{e}_{1}-\Phi(t) G_{15} M_{55}^{-1} \hat{e}_{5}, \\
-\Phi(t) G_{15} M_{55}^{-1} \hat{\tau} \leq-\left.1_{(N \times 1)} f_{r 1}\right|_{\min }-\Phi(t) \hat{e}_{1}+\Phi(t) G_{15} M_{55}^{-1} \hat{e}_{5},
\end{gathered}
$$

though this effect will not be explored in this work.

\section{Results from Pseudo-Spectral Optimal Control}

\subsection{Performance Sensitivity to Penalty Weight}

The two contributions to the objective function in Eqn. (61) are not of the same units, and the interrelationship between them is complex. To explore the effect on device performance, each geometric configuration was optimized throughout the wave period range of $5 \mathrm{~s}-20 \mathrm{~s}$ while varying the penalty weight. A sample set of results is plotted in Fig. 6, which highlights the performance of the optimization routine compared to the passive and maximum constrained values. The penalty weight that matched the passive TAP, $L_{B}$, and the maximum constrained TAP, $U_{B}$, were 
collected for each geometric configuration and are plotted in Fig. 6(d). The results show a significant variation across the range of wave frequencies and between geometries. The results in Fig. 6(a) verifies that the optimization routine is achieving the desired results when considering the extremes of the tested penalty weights. At the smallest penalty weights the capture width is equal to the known maximum constrained value whereas at the largest penalty weights the minimization of the surge-foundation force takes precedent and is zero. Since the wave period is lower than the 2-flap geometry resonance period, the reduction in surge-foundation force is followed by a similar reduction in PTO control torque, see Fig. 6(b); however, the torque amplitude will never reach zero for this wave condition in order to eliminate the surge-foundation load. It was found in Eidsmoen (1996) that greater TAP could be absorbed when allowing for nonsinusoidal motion, but this was followed by greater WEC velocities and PTO loads, and because this work is focused on reducing these peak values this range will not be considered.

The intermediate range of the penalty weights provide far more interesting results because a faster decrease in the surge-foundation force amplitude is observed than compared to the capture efficiency. This is best viewed in the bottom left plot of Fig. 6(b), which shows the ratio of optimized capture efficiency, $C_{w}$, and surge-foundation load, $\left|X_{r 1}\right|$, compared to the maximum constrained value as a function of the penalty weight. There is a distinct penalty weight that leads to a reduction in capture efficiency of $40 \%$, whereas the reduction in surge load is $50 \%$. This corresponds to the best power-to-force point at which an increase in capture width requires a greater proportionate increase in surge-foundation force. The optimal power-to-force point occurs slightly before the slope of the reactive power reaches its greatest growth. The optimization routine currently assumes perfect efficiency of the PTO unit in both the motor and generator configurations, though it is well known that the bidirectional efficiencies are significant when the energy flow is reversible in application (Genest et al., 2014). After this point the gain in net power can be at most increased by approximately $60 \%$, whereas the corresponding increase in reactive power is $520 \%$ and PTO control torque by $225 \%$, see Fig. 6(b). For each geometry and wave period the optimal benefit-to-cost point was calculated, and the results are plotted in Fig. 7. These results can be considered optimum in the sense that the power-to-load ratio is maximized; however, consideration for the required reactive power is not included in the optimization and limits the feasible application. In Fig. 7(a), there are instances when the reactive power requirement is greater than the net power output. It is the opinion of the authors that these conditions are not practical, and thus there are specific operating ranges that are appropriate to use the control techniques discussed in this document. The best operating range for the 4-flap geometry is between $0.8 \mathrm{rad} / \mathrm{s}-1.05 \mathrm{rad} / \mathrm{s}$, the 3 -flap geometry between $0.6 \mathrm{rad} / \mathrm{s}-0.8 \mathrm{rad} / \mathrm{s}$, the 2 -flap geometry between $0.4 \mathrm{rad} / \mathrm{s}-0.6 \mathrm{rad} / \mathrm{s}$, and for the 1 -flap geometry below $0.4 \mathrm{rad} / \mathrm{s}$ while above $1.05 \mathrm{rad} / \mathrm{s}$. The separate range for the 1-flap geometry occurs from the resonance regime at the edge of the low-frequency regime and the high-frequency regime when the TAP is linearly proportional to the pitch wave-excitation torque for highly constrained motion. Another benefit of the operating windows is the minimization of the required PTO control torque, as plotted in Fig. 7(b). This leads to a reduction in cost associated with the selection of a smaller PTO unit and lower fatigue loads that become of greater importance when considering the lifetime of the device.

\subsection{Optimal Control Time History}

The effect of the penalty weight on the time history of the controller has been plotted in Fig. 8. Four penalty weights have been selected to illustrate the transition from the maximum TAP to near elimination of the surgefoundation load. As the penalty weight is reduced, the PTO control torque ensures that the pitch angular velocity remains in phase with the pitch wave-exciting torque while staying within the pitch displacement limits, see Fig. 8(a). This is accompanied by the greatest PTO control torque, surge-foundation force, and standard deviation in instantaneous power. As the penalty weight is increased, the controller first maintains near optimum phase while reducing the amplitude of motion; however, eventually a greater phase shift is introduced by the controller to eliminate a greater proportion of the surge-foundation force; refer to Eqn. (35). The larger reduction in surge-foundation force compared to the TAP is due to the phase difference between the PTO control torque and pitch angular velocity. As shown in Fig. 8(b), when the penalty weight is increased the time of the peak pitch angular velocity moves back in time and the peak in PTO control torque moves forward in time, see Fig. 8(d). The phase shift in pitch angular velocity assists in reducing the surge-foundation force while at the same time the moving closer in phase with the PTO control torque. As the phase difference is reduced, the reactive power and instantaneous power peaks also are reduced as shown in Fig. 8(c) and Fig. 8(e) respectively. When there is no phase difference between the PTO control torque and pitch angular velocity, the reactive power component is eliminated and the maximum passive performance is obtained as this corresponds to the use of an optimum linear PTO-damping coefficient. The swings in bi-directional energy flow 


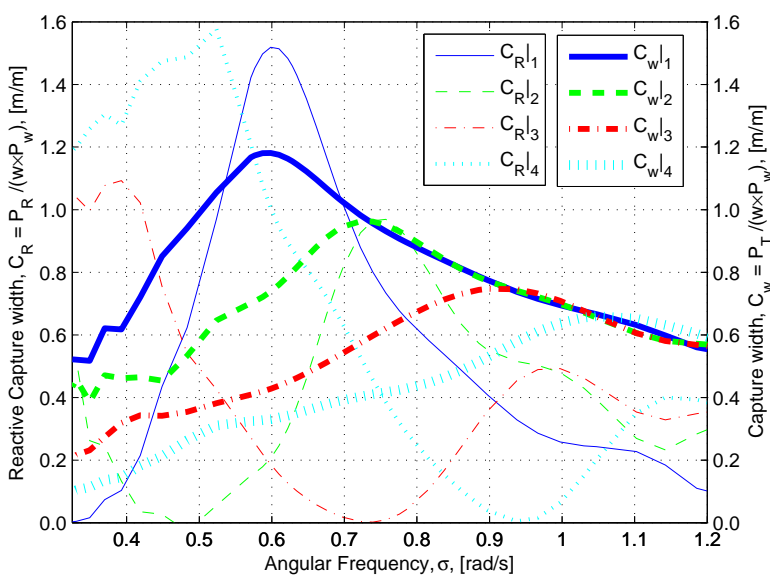

(a) Reactive \& Net Capture Width at Optimum Power-to-Force

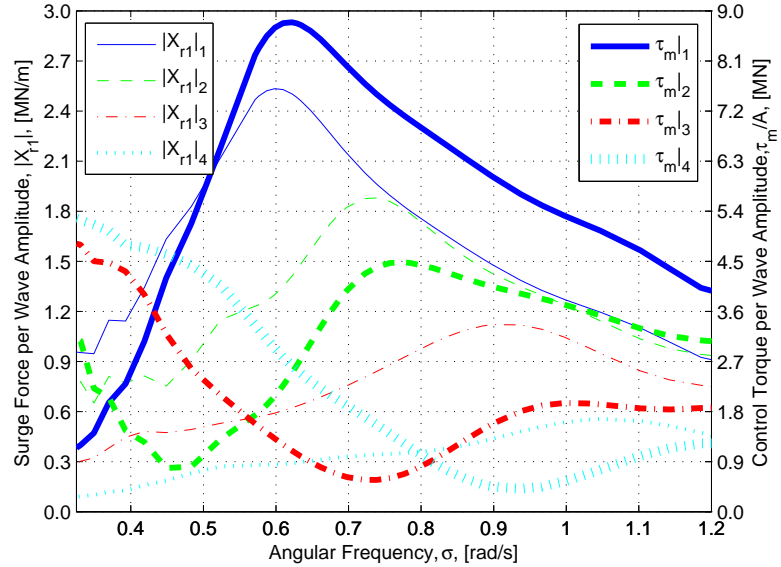

(b) Surge-Foundation Force \& PTO Torque at Optimum Power-to-Force

Figure 7. Optimum power-to-force values for each geometric configuration with a maximum pitch displacement of $\pi / 6$ and a wave amplitude of $0.125 \mathrm{~m}$. The performance values were obtained at each frequency and for each geometry at the given penalty weight that provided the greatest reduction in the surge-foundation force compared to TAP, denoted by the markers in Fig. 6(c).

are highlighted in Fig. 8(f) which dwarfs the fluctuations from the passive performance. By comparing the maximum constrained and $\gamma=10^{-6.5}$ time histories, the TAP can be doubled but requires a five fold increase in peak instantaneous power. Thus, the increase in PTO effort can outpace the TAP when attempting to collect the remaining available wave power. Furthermore, the required PTO control torque amplitude to obtain the maximum constrained TAP is four times greater than the pitch wave-exciting torque; however, torque is not invariant to the wave amplitude, and limits on the PTO output will lead to reductions in power absorption in larger sea states. If a limit on the amplitude of the control torque is set equal to the amplitude of the pitch wave-exciting torque, then the TAP would be limited to approximately half of the constrained maximum.

\section{Conclusion}

This paper has used pseudo-spectral optimal control to evaluate the regular wave performance of a novel WEC concept that combines an OSWEC with active geometry. The active geometry provides an additional degree-offreedom that can be used to alter the hydrodynamic properties which, in addition to the PTO, can be used for greater control of device performance. The analysis first provided maximum and minimum bounds on the TAP and the resulting surge-foundation force. For the maximum bound, it was assumed that the device motion was constrained, but it remained sinusoidal, and optimum phase was maintained between the pitch wave-exciting torque and the pitch angular velocity. The lower bound was obtained by assuming that the PTO unit could provide only a linear resistive damping torque that was tuned to the incident wave leading to passive control. The equations of motion were constructed assuming linear hydrodynamic theory, though extension to include nonlinear effects can be easily realized (Bacelli and Ringwood, 2014). This work has highlighted issues that arise when WEC control focuses solely on maximizing power absorption as it is accompanied by greater structural and PTO loads that will lead to a higher levelized cost of energy.

The optimal control problem was modified to include the squared sum of the surge-foundation force in the objective function. This is in contrast to the optimal control problem defined solely by power absorption (Herber and Allison, 2013 and Bacelli and Ringwood, 2015). The optimal solution can now be tuned based on the penalty weight magnitude to emphasize either power absorption or load mitigation. Verification of the optimization problem began by evaluating its performance as the surge foundation force penalty weight was varied. It was shown that as $\gamma \rightarrow 0$ the maximum constrained power was recovered, whereas when $\gamma \rightarrow \infty$ the surge-foundation force was completely eliminated. The results obtained in the intermediate range of the penalty weights were of greater interest as it lead to a better understanding of the power-to-load relationships. It was found that the surge-foundation load decreased at a faster rate than time-averaged absorbed power, leading to a penalty weight that provided the greatest power-to-load 


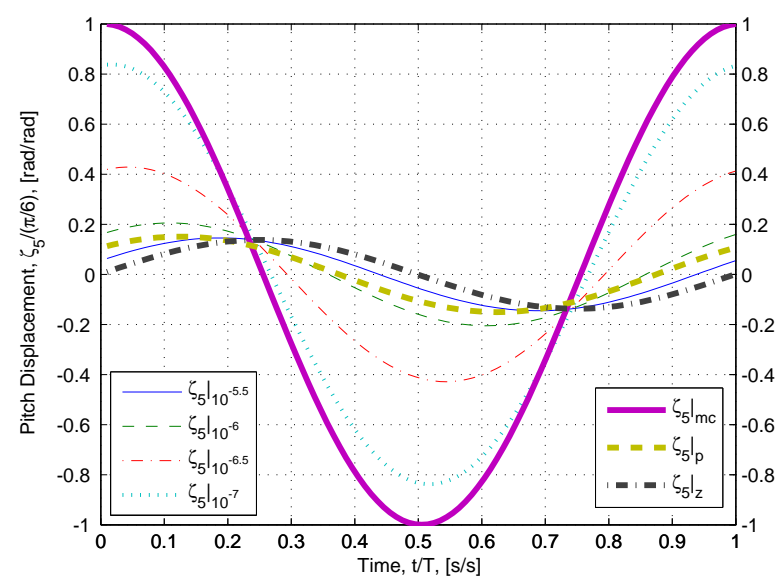

(a) Pitch displacement, $\zeta_{5}$.

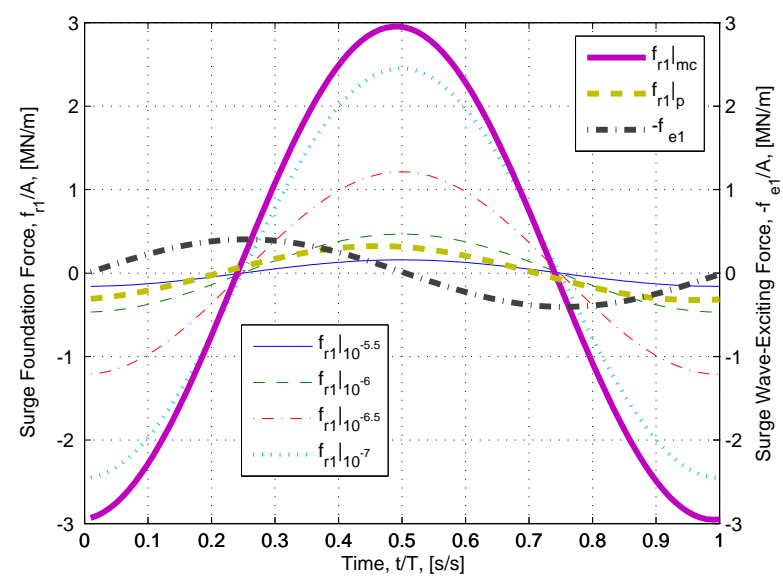

(c) Surge-foundation force, $f_{r 1}$, and surge wave-exciting force, $f_{e 1}$.

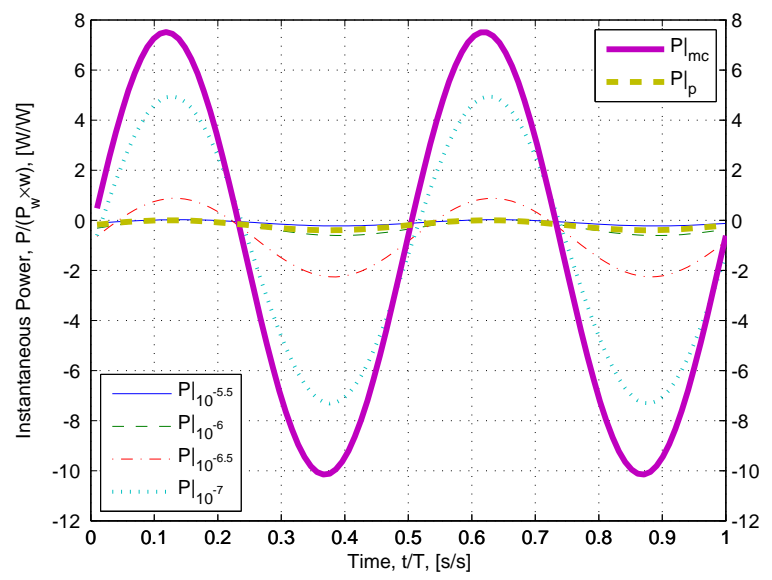

(e) Instantaneous power, $P=\dot{\zeta}_{5} \tau_{m}$.

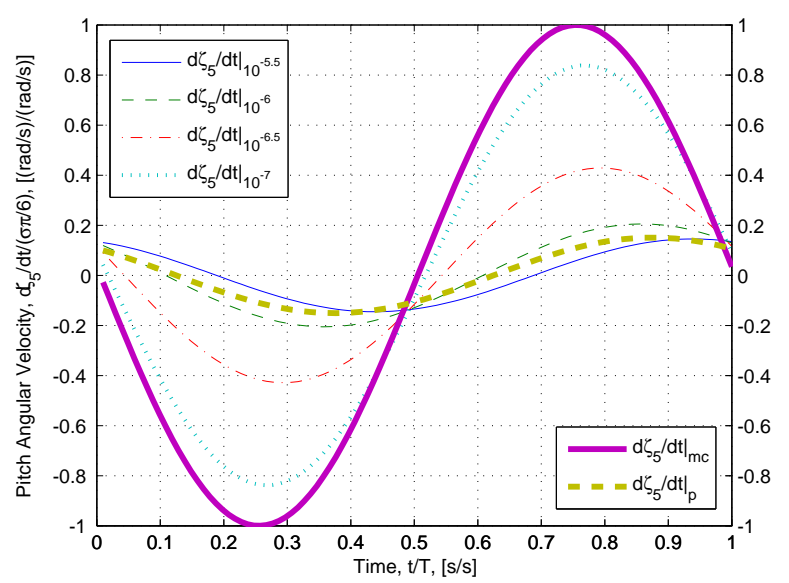

(b) Pitch angular velocity, $\dot{\zeta}_{5}$.

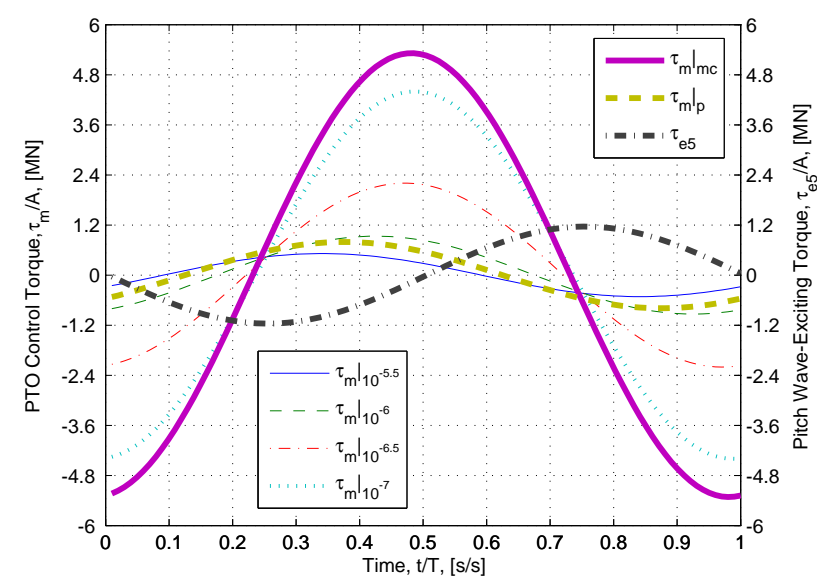

(d) PTO control torque, $\tau_{m}$, and pitch wave-exciting torque, $\tau_{e 5}$.

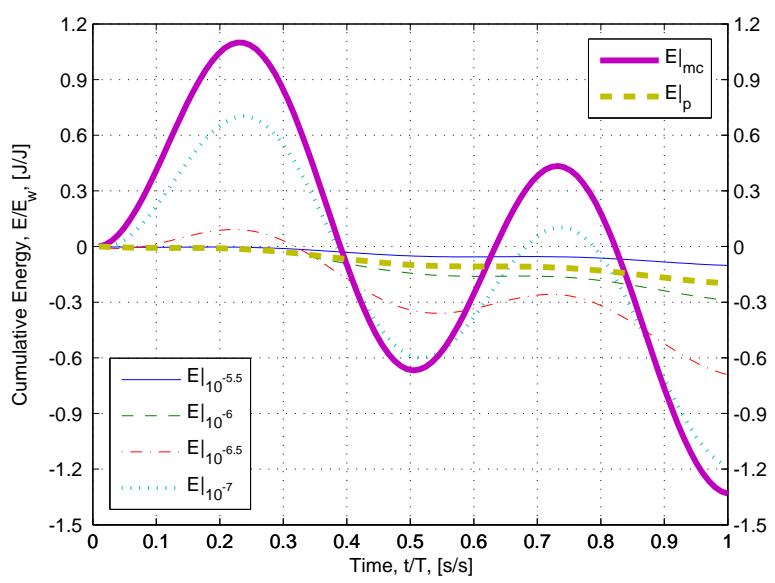

(f) Cumulative energy, $E=\int_{0}^{t} \dot{\zeta}_{5} \tau_{m} d t$.

Figure 8. Time history of performance metrics for the 2-flap geometric configuration with a pitch-displacement amplitude limit of $\pi / 6$, a wave amplitude of $0.125 \mathrm{~m}$, and a wave period of $10 \mathrm{~s}$, while the penalty weight, $\gamma$, has been varied. The subscript $\mathrm{mc}_{\mathrm{c}}$ denotes performance for maximum constrained power as given by Eqns. (25)-(29). The subscript ${ }_{p}$ denotes performance for passive power as given by Eqns. (16)-(19). The subscript $z$ denotes performance when the pitch displacement follows the amplitude and phase set by Eqn. (35)-(36) to eliminate the surge-foundation force. The quantity $E_{w}$ denotes the cumulative absorbed energy assuming a nondimensional capture width of 1 . 
ratio. However, the optimum penalty weight was found to have significant variation based on the incident wave frequency and WEC geometry. As the penalty weight was increased the optimizer shifted away from optimum phase and reduced the pitch-displacement amplitude. Furthermore, the reactive power contribution was reduced when increasing the penalty weight as the phase difference between the pitch angular velocity and PTO control torque was eliminated. The pseudo-spectral techniques described in this paper can be solved very efficiently because of the convexity of the objective function, which is a result of the linear nature of the equation of motion. The addition of nonlinear dynamics and constraints will increase the computation time because the problem is no longer convex and the solution can be susceptible to local minimums.

\section{Acknowledgments}

This work was supported by the U.S. Department of Energy under Contract No. DE-AC36-08GO28308 with the National Renewable Energy Laboratory. Funding for the work was provided by the DOE Office of Energy Efficiency and Renewable Energy, Wind and Water Power Technologies Office.

\section{References}

Abraham, E., Kerrigan, E.C., 2013. Optimal active control and optimization of a wave energy converter. IEEE Transactions on Sustainable Energy 4 (2), 324-332.

Babarit, A., Clément, A.H., 2006. Optimal latching control of a wave energy device in regular and irregular waves. Applied Ocean Research 28 , 77-91.

Babarit, A., Guglielmi, M., Clément, A.H., 2009, Declutching control of a wave energy converter. Ocean Engineering 36 (12), $1015-1024$.

Babarit, A., Hals, J., Muliwan, M.J., Moan, T., Krokstad, J., 2012. Numerical benchmarking study of a selection of wave energy converters. Renewable Energy 41, 44-63.

Bacelli, G., Ringwood, J.V., 2011. A Control System for a Self-reacting Point Absorber Wave Energy Converter Subject to Constraints. In: 18th World Congress of the International Federation of Automatic Control, Milano, Italy,11387-11392.

Bacelli, G., Ringwood, J.V., 2014. Nonlinear Optimal Wave Energy Converter Control with Application to a Flap-type Device. In: 19th World Congress of the International Federation of Automatic Control, Cape Town, South Africa, 7696-7701.

Bacelli, G., Ringwood, J.V., 2015. Numerical Optimal Control of Wave Energy Converters. IEEE Transactions on Sustainable Energy 6 (2), $133-145$.

Cretel, J.A.M, Lightbody, G., Thomas, G.P., Lewis, A.W., 2011. Maximisation of energy capture by a wave-energy point absorber using model predictive control. In: 18th World Congress of the International Federation of Automatic Control, Milano, Italy, 3714-3721.

Cummins, W.E., 1962. The impulse response function and ships motions. Schiffstechnik 9, 101-109.

Eidsmoen, H., 1996. Optimum control of a floating wave-energy converter with restricted amplitude. Journal of Offshore Mechanics and Arctic Engineering $118(2), 96-102$.

Evans, D.V., 1976. A theory for wave-power absorption by oscillating bodies. Journal of Fluid Mechanics 7 (1), 1-25.

Evans, D.V., 1981. Maximum wave-power absorption under motion constraints. Applied Ocean Research 3 (4), 200-203.

Falnes, J., 2002. Optimum control of oscillation of wave-energy converters. International Journal of Offshore and Polar Engineering 12 (2), $147-$ 154.

Flocard, F., Finnigan, T.D., 2012. Increasing power capture of a wave energy device by inertia adjustment. Applied Ocean Research 34, $126-134$.

Garcia-Rosa, P.B., Bacelli, G., and Ringwood, J.V., 2015. Control-informed geometric optimization of wave energy converters: The impact of device motion and force constraints. Energies 8, 13672-13687.

Genest, R., Félicien, B., Clément, A.H., Babarit A., 2014. Effect of non-ideal power take-off on the energy absorption of a reactively controlled one degree of freedom wave energy converter. Applied Ocean Research 48, 236-243.

Gomes, R.P.F., Lopes, M.F.P., Henriques, J.C.C., Gato, L.M.C, Falcão, A.F.O., 2015. The dynamics and power extraction of bottom-hinged plate wave energy converters in regular and irregular waves. Ocean Engineering 96, 86-99.

Hals, J., Bjarte-Larsson, T., Falnes, J., 2002. Optimum reactive control and control by latching of a wave-absorbing semisubmerged heaving sphere. In: 21st International Conference on Offshore Mechanics and Arctic Engineering, Oslo, Norway, pp. 415-423.

Hals, J., Falnes, J., Moan, T., 2011. Constrained optimal control of a heaving buoy wave-energy converter. Journal of Offshore Mechanics and Arctic Engineering 133 (1), 011401.

Herber, D.R., Allison, J.T., 2013. Wave energy extraction maximization in irregular ocean waves using pseudospectral methods. In: ASME International Design Engineering Technical Conferences and Computers and Information in Engineering Conference, Portland, Oregon, USA.

Kurniawan, A., Moan, T., 2012. Characteristics of a pitching wave absorber with rotatable flap. Energy Procedia 20, $134-147$.

Li, G., Belmont, M.R., 2014. Model predictive control of sea wave energy converters —Part I: A convex approach for the case of a single device. Renewable Energy 69, 453-463.

Lucas, J., Livingstone, M., Vuorinen, M., Cruz, J., 2012. Development of a wave energy converter (WEC) design tool - application to the WaveRoller WEC including validation of numerical estimates. In: Fourth International Conference on Ocean Energy, Dublin, Ireland.

Musial, W., Lawson, M., Rooney, S., 2013. Marine hydrokinetic technology (MHK) instrumentation, measurement, and computer modeling workshop. National Renewable Energy Laboratory, Technical Report NREL/TP-5000-57605. 
Ogilvie, T., 1964. Recent progress towards the understanding and prediction of ship motions. In: Fifth Symposium on Naval Hydrodynamics, Bergen, Norway, pp. 3-79.

Pecher, A., Kofoed J.P., Espedal, J., Hagberg, S., 2010. Results of an experimental study of the Langlee wave energy converter. In: 20th International Offshore and Polar Engineering Conference, Beijing, China, pp. 877-885.

Pecher, A., Kofoed, J.P., Larsen, T., Marchalot, T., 2012. Experimental study of the Weptos wave energy converter. In: 31st International Conference on Ocean, Offshore, and Arctic Engineering, Rio de Janeiro, Brazil.

Ramudu, E., 2011. Ocean wave energy-driven desalination systems for off-grid coastal communities in developing countries. In: IEEE Global Humanitarian Technology Conference, Seattle, Washington, USA, pp. 1129-1142.

Siegel, S.G., Fagley, C., Romer, M., McLaughlin, T.E., 2011. Experimental wave cancellation using a cycloidal wave energy converter. In: Ninth European Wave and Tidal Energy Conference, Southampton, UK.

Tom, N., Lawson, M.J., Yu, Y.H., Wright, A.D., 2016a. Spectral modeling of an oscillating surge wave energy converter with active control surfaces. Applied Ocean Research 56, 143-156.

Tom, N., Lawson, M.J., Yu, Y.H., Wright, A.D., 2016b. Development of a nearshore oscillating surge wave energy converter with variable geometry. Renewable Energy 96, 410-424.

Whittaker, T., Folley, M., 2012. Nearshore oscillating wave surge converters and the development of Oyster. Philosophical Transactions Royal Society A. 370, 345-364.

WAMIT Version 7.0 User Manual, http://www.wamit.com; 2013.

Zurkinden, A.S., Lambertsen, S.H., Damkilde, L., Gao, Z., Moan T., 2013. Fatigue analysis of a wave energy converter taking into account different control strategies," In: 32nd ASME International Conference on Ocean, Offshore, and Arctic Engineering, Nantes, France.

\section{Appendix A. Matrix Representation of Convolution Integrals}

The expression given in Eqn. (12) can be expanded to keep the real component:

$$
\tau_{r 55 c}(t)=\left[\sigma^{2}\left(\mu_{55}(\infty)-\mu_{55}(\sigma)\right)\right]\left[\mathfrak{R}\left\{\xi_{5}\right\} \cos \sigma t-\mathfrak{J}\left\{\xi_{5}\right\} \sin \sigma t\right]-\sigma \lambda_{55}(\sigma)\left[\mathfrak{R}\left\{\xi_{5}\right\} \sin \sigma t+\mathfrak{J}\left\{\xi_{5}\right\} \cos \sigma t\right],
$$

which can be expressed as a matrix multiplication as follows:

$$
\begin{gathered}
\tau_{r 55 c}(t)=\Phi(t) G \hat{\xi} \\
\Phi(t)=[\cos \sigma t, \sin \sigma t], G=\left[\begin{array}{cc}
\sigma^{2}\left[\mu_{55}(\infty)-\mu_{55}(\sigma)\right] & \sigma \lambda_{55}(\sigma) \\
-\sigma \lambda_{55}(\sigma) & \sigma^{2}\left[\mu_{55}(\infty)-\mu_{55}(\sigma)\right]
\end{array}\right], \quad \hat{\xi}=\left[\begin{array}{c}
\mathfrak{R}\left\{\xi_{5}\right\} \\
-\mathfrak{J}\left\{\xi_{5}\right\}
\end{array}\right] .
\end{gathered}
$$

The expression can also be written in terms of the pitch angular velocity, which has been shown to reduce the number of coefficients that must be solved. The expansion of Eqn. (12) now becomes:

$$
\tau_{r 55 c}(t)=\lambda_{55}(\sigma)\left[\mathfrak{R}\left\{\dot{\xi}_{5}\right\} \cos \sigma t-\mathfrak{J}\left\{\dot{\xi}_{5}\right\} \sin \sigma t\right]+\left[\sigma\left(\mu_{55}(\infty)-\mu_{55}(\sigma)\right)\right]\left[\mathfrak{R}\left\{\dot{\xi}_{5}\right\} \sin \sigma t+\mathfrak{J}\left\{\dot{\xi}_{5}\right\} \cos \sigma t\right],
$$

which is represented in the same form as in Eqn. (A.2) with changes to the following matrices:

$$
G_{55}=\left[\begin{array}{cc}
\lambda_{55}(\sigma) & -\sigma\left[\mu_{55}(\infty)-\mu_{55}(\sigma)\right] \\
\sigma\left[\mu_{55}(\infty)-\mu_{55}(\sigma)\right] & \lambda_{55}(\sigma)
\end{array}\right], \hat{\psi}=\left[\begin{array}{c}
\mathfrak{R}\left\{\dot{\xi}_{5}\right\} \\
-\mathfrak{J}\left\{\dot{\xi}_{5}\right\}
\end{array}\right] .
$$

With additional manipulation the wave-radiation convolution integral can be represented as:

$$
\begin{gathered}
\tau_{r 55}(t)=\Phi(t)\left(G_{55}-\mu_{55}(\infty) \Gamma\right) \hat{\psi} \\
G_{55}=\left[\begin{array}{cc}
\lambda_{55}(\sigma) & \sigma \mu_{55}(\sigma) \\
-\sigma \mu_{55}(\sigma) & \lambda_{55}(\sigma)
\end{array}\right], \Gamma=\left[\begin{array}{cc}
0 & \sigma \\
-\sigma & 0
\end{array}\right] .
\end{gathered}
$$

The excitation convolution integral can be represented using a similar matrix form:

$$
\begin{aligned}
& f_{e 5}(t)=\Phi(t) \hat{e}_{5}, \\
& \hat{e}_{5}=\left[\begin{array}{c}
A \mathfrak{R}\left\{X_{5}\right\} \\
-A \mathfrak{J}\left\{X_{5}\right\}
\end{array}\right] .
\end{aligned}
$$

